Pacific

Journal of

Mathematics

\title{
REPRESENTATION THEORY OF TYPE B AND C STANDARD LEVI $W$-ALGEBRAS
}

JONATHAN BROWN AND SIMON M. GOODWIN 


\title{
REPRESENTATION THEORY OF TYPE B AND C STANDARD LEVI $W$-ALGEBRAS
}

\author{
JONATHAN BROWN AND Simon M. GOODWIN
}

\begin{abstract}
We classify the finite-dimensional irreducible representations with integral central character of finite $W$-algebras $U(\mathfrak{g}, e)$ associated to standard Levi nilpotent orbits in classical Lie algebras of types $B$ and $C$. This classification is given explicitly in terms of the highest weight theory for finite $W$-algebras.
\end{abstract}

\section{Introduction}

Let $e$ be a nilpotent element in the Lie algebra $\mathfrak{g}$ of a reductive algebraic group $G$ over $\mathbb{C}$. The finite $W$-algebra $U(\mathfrak{g}, e)$ associated to the pair $(\mathfrak{g}, e)$ is an associative algebra obtained from $U(\mathfrak{g})$ by a certain quantum Hamiltonian reduction. There has been a great deal of recent interest in finite $W$-algebras and their representation theory; for an overview, see the survey article [Losev 2011b].

In [Brown and Goodwin 2013a; 2013b], we gave a combinatorial classification of the finite-dimensional irreducible $U(\mathfrak{g}, e)$-modules, where $\mathfrak{g}$ is a classical Lie algebra and $e$ is an even-multiplicity nilpotent element; we recall that $e$ is said to be even multiplicity if all parts of the Jordan type of $e$ occur with even multiplicity. This classification is given in terms of the highest weight theory for finite $W$-algebras from [Brundan et al. 2008].

Now recall that a nilpotent element $e$ of $\mathfrak{g}$ is said to be of standard Levi type if $e$ is in the regular nilpotent orbit of a Levi subalgebra of $\mathfrak{g}$. It is easy to check that if $\mathfrak{g}$ is of classical type and $e$ is even multiplicity, then $e$ is standard Levi. In this paper, we extend the results of [Brown and Goodwin 2013a] to classify the finite-dimensional irreducible $U(\mathfrak{g}, e)$-modules with integral central character, where $\mathfrak{g}$ is of type $\mathrm{B}$ or $\mathrm{C}$ and $e$ is any standard Levi nilpotent element; see Theorem 1.2. We plan to deal with the case of any (not necessarily integral) central characters in future work, where different methods will be required. We recall (see, for example, the footnote to [Premet 2007, Question 5.1]) that the centre of $U(\mathfrak{g}, e)$ is canonically identified with the centre of $U(\mathfrak{g})$, which allows one to define integral central characters.

The situation for $\mathfrak{g}$ of type $\mathrm{D}$ and $e$ standard Levi, but not even-multiplicity, is more awkward. In this case the combinatorics become more complicated and the

MSC2010: 17B10, 81R05.

Keywords: $W$-algebras, representation theory. 
statement of the classification of finite-dimensional irreducible $U(\mathfrak{g}, e)$-modules cannot be given simply in terms of a row-equivalent to column-strict condition as in Theorem 1.2.

We remark here that finite $W$-algebras corresponding to nilpotent elements of standard Levi type are a natural class to consider. This is because such finite $W$ algebras are particularly amenable to the highest weight theory from [Brundan et al. 2008], as explained in Section 2C.

Losev and Ostrik [2013] have achieved a classification of the finite-dimensional $U(\mathfrak{g}, e)$-modules of integral central character for any reductive Lie algebra $\mathfrak{g}$ in the following manner: Losev [2010] gave a surjection from the primitive ideals of finite codimension of $U(\mathfrak{g}, e)$ to the primitive ideals of $U(\mathfrak{g})$ having associated variety equal to the closure $\overline{G \cdot e}$ of the $G$-orbit of $e$. There is a natural action of the component group $C$ of the centralizer of $e$ in $G$ on the set of primitive ideals of $U(\mathfrak{g}, e)$, as explained, for example, in the introduction to [Losev 2011a]. This last paper extends the results of [Losev 2010] to show that the fibres of the above surjection are precisely $C$-orbits. The classification in [Losev and Ostrik 2013] is accomplished by describing the fibres of this map, i.e., determining the stabiliser of the $C$-orbit for each fibre. The primitive ideals with associated variety equal to $\overline{G \cdot e}$ can be described thanks to the methods of a variety of mathematicians in the 1970s and 1980s; see for example [Jantzen 1983] and the references therein for details.

We go on to explain the results of this paper in more detail, so we take $\mathfrak{g}$ to be of type B or C; that is, $\mathfrak{g}=\mathfrak{s o}_{2 n+1}$ or $\mathfrak{g}=\mathfrak{s p}_{2 n}$ for some $n \in \mathbb{Z}_{\geq 2}$. We recall that nilpotent orbits in $\mathfrak{g}$ are parametrized by their Jordan type. Thus they are given by partitions of $2 n+1$ (respectively $2 n$ ) where all even (respectively odd) parts occur with even multiplicity when $\mathfrak{g}=\mathfrak{s o}_{2 n+1}$ (respectively $\mathfrak{g}=\mathfrak{s p}_{2 n}$ ). In this paper we consider only nilpotent orbits which are standard Levi but not even-multiplicity, as the latter are dealt with in [Brown and Goodwin 2013a; 2013c]. This means that the Jordan type of $e$ is given by a partition of the form

$$
\boldsymbol{p}=\left(p_{1}^{2 a_{1}}<p_{2}^{2 a_{2}}<\cdots<p_{d-1}^{2 a_{d-1}}<p_{d}^{2 a_{d}+1}<p_{d+1}^{2 a_{d+1}}<\cdots<p_{r}^{2 a_{r}}\right) ;
$$

that is, all parts of $\boldsymbol{p}$ occur with even multiplicity except for one part $p_{d}$, which occurs with odd multiplicity. This description of partitions corresponding to standard Levi nilpotent orbits follows, for example, from the explicit description of Levi subgroups regular nilpotent elements given in [Jantzen 2004, §4.5, §6.3]. It will be more convenient for us to reindex this partition and write it as

$$
\boldsymbol{p}=\left(p_{1}^{2} \leq p_{2}^{2} \leq \cdots \leq p_{d-1}^{2}<p_{0} \leq p_{d}^{2} \leq \cdots \leq p_{r}^{2}\right) .
$$

In this paper, we only consider finite-dimensional irreducible representations 
for $U(\mathfrak{g}, e)$ with integral central character. As we explain in Section $2 \mathrm{C}$, such representations occur only when $e$ is a special nilpotent element in the sense of [Lusztig 1979]. In terms of the partition $\boldsymbol{p}$, this means that the dual partition of $\boldsymbol{p}$ is the Jordan type of a nilpotent orbit in $\mathfrak{g}$. Explicitly, this means that $p_{i}$ must be odd for all $i \geq d$ when $\mathfrak{g}=\mathfrak{s o}_{2 n+1}$ or $p_{i}$ must be even for all $i \leq d$ when $\mathfrak{g}=\mathfrak{s p}_{2 n}$. This can be deduced from the description of special symbols in [Lusztig 1979] and [Barbasch and Vogan 1982, Theorem 18]; see also [Collingwood and McGovern 1993, Proposition 6.3.7]. For the remainder of the paper we assume that $\boldsymbol{p}$ is a partition satisfying these conditions.

We use symmetric pyramids to describe much of the combinatorics underlying $U(\mathfrak{g}, e)$-modules. The symmetric pyramid for $\boldsymbol{p}$, denoted by $P$, is a finite connected collection of boxes in the plane such that

- the boxes are arranged in connected rows;

- the boxes are symmetric with respect to both the $y$-axis and the $x$-axis;

- each box is 2 units by 2 units;

- the lengths of the rows from top to bottom are given by

$$
p_{1} \ldots, p_{r}, p_{0}, p_{r}, \ldots, p_{1} \text {. }
$$

An $s$-table with underlying symmetric pyramid $P$ is a skew-symmetric (with respect to the origin) filling of $P$ with complex numbers. We define $\operatorname{sTab}(P)$ to be a certain set of s-tables depending on whether $\mathfrak{g}=\mathfrak{s o}_{2 n+1}$ or $\mathfrak{s p}_{2 n}$. For $\mathfrak{g}=\mathfrak{s p}_{2 n}$ we let sTab $(P)$ denote the set of s-tables with underlying symmetric pyramid $P$ such that all entries are integers, whereas for $\mathfrak{g}=\mathfrak{s o}_{2 n+1}$ we define $\operatorname{sTab}(P)$ to be the s-tables such that either all entries are in $\mathbb{Z}$ or all entries are in $\frac{1}{2}+\mathbb{Z}$. Let sTab $\leq(P)$ denote the elements of $\operatorname{sTab}(P)$ that have nondecreasing rows. As explained in Section $3 \mathrm{C}$, the elements of $\mathrm{sTab}^{\leq}(P)$ parametrize the irreducible highest weight $U(\mathfrak{g}, e)$-modules; given $A \in \operatorname{sTab}(P)$, we write $L(A)$ for the corresponding irreducible highest weight $U(\mathfrak{g}, e)$-module.

An example of an s-table in $\operatorname{sTab}^{\leq}(P)$, when $\mathfrak{g}=\mathfrak{s p}_{2 n}, \boldsymbol{p}=\left(2^{2}, 4,5^{2}\right)$ and $P$ is the symmetric pyramid for $\boldsymbol{p}$, is this:

\begin{tabular}{|l|l|l|l|l|l|}
\cline { 2 - 5 } \multicolumn{1}{c|}{} & 6 & \multicolumn{1}{c|}{7} & \multicolumn{2}{c}{} \\
\hline 2 & 4 & 5 & 8 & 9 \\
\hline-3 & -1 & 1 & 3 & \\
\hline-9 & -8 & -5 & -4 & -2 \\
\hline \multicolumn{7}{|c|}{} & -7 & -6 & \\
\hline
\end{tabular}


The left justification of an s-table is the diagram created by left-justifying all of the s-table's rows. We say an s-table is justified row-equivalent to column-strict if the row equivalence class of its left justification contains a table in which every column is strictly decreasing; we note that there can be a gap in the middle of some columns, and we require entries to be strictly decreasing across this gap. We write $\operatorname{sTab}^{c}(P)$ for the set of all $A \in \operatorname{sTab}(P)$ that are justified row-equivalent to column-strict. It is easy to see that the example of the s-table above is an element of $\operatorname{sTab}^{c}(P)$.

Recall that $C$ denotes the component group of the centralizer of $e$ in $G$. In Section 7A, we define an action of $C$ on the subset of $\mathrm{sTab}^{\leq}(P)$ corresponding to finite-dimensional $U(\mathfrak{g}, e)$-modules.

Now we can state the main theorem of this paper:

Theorem 1.2. Let $\mathfrak{g}=\mathfrak{s o}_{2 n+1}$ or $\mathfrak{s p}_{2 n}$, let $\boldsymbol{p}$ be a partition corresponding to a standard Levi special nilpotent orbit in $\mathfrak{g}$, let $e$ be an element of this orbit and let $P$ be the symmetric pyramid for $\boldsymbol{p}$. Then

$$
\left\{L(A) \mid A \in \operatorname{sTab}^{\leq}(P), A \text { is } C \text {-conjugate to some } B \in \operatorname{sTab}^{c}(P)\right\}
$$

is a complete set of isomorphism classes of finite-dimensional irreducible $U(\mathfrak{g}, e)$ modules with integral central character. Moreover, the $C$-action on s-tables agrees with the $C$-action on finite-dimensional irreducible $U(\mathfrak{g}, e)$-modules.

Analogous results to [Brown and Goodwin 2013a, Corollaries 5.17 and 5.18] hold in the present situation. So when all parts of $\boldsymbol{p}$ have the same parity, if $L(A)$ is finite-dimensional, then, in fact, $A$ is row-equivalent to column-strict as an stable. Thus in this case $L(A)$ can be obtained as a subquotient of the restriction of a finite-dimensional $U(\mathfrak{g}(0))$-module via the Miura map. We refer the reader to the discussion before Corollary 5.18 in that reference for more details, and to Section $2 \mathrm{~A}$ below for the definition of $\mathfrak{g}(0)$.

Theorem 1.2 and the correspondence of finite-dimensional irreducible $U(\mathfrak{g}, e)$ modules and primitive ideals of $U(\mathfrak{g})$ with associated variety $\overline{G \cdot e}$ discussed above allow us to deduce the following corollary. It gives an explicit description of the primitive ideals of $U(\mathfrak{g})$ having associated variety equal to $\overline{G \cdot e}$ and integral central character. A method to classify these primitive ideals was originally given in [Barbasch and Vogan 1982]. In the corollary, $L\left(\lambda_{A}\right)$ denotes the irreducible highest weight $U(\mathfrak{g})$-module defined from an s-table $A$ as explained in Section $3 \mathrm{C}$ below.

Corollary 1.3. The set of primitive ideals with integral central character and associated variety $\overline{G \cdot e}$ is equal to

$$
\left\{\operatorname{Ann}_{U(\mathfrak{g})} L\left(\lambda_{A}\right) \mid A \in \operatorname{sTab}^{c}(P) \cap \operatorname{sTab}^{\leq}(P)\right\} .
$$

Below we give an outline of the proof of Theorem 1.2. 
The key step is to deal with the case where $\boldsymbol{p}$ has three parts. We deal with this case using the relationship between finite-dimensional irreducible representations of $U(\mathfrak{g}, e)$ and primitive ideals of $U(\mathfrak{g})$ with associated variety equal to $\overline{G \cdot e}$. Using this and results of Barbasch and Vogan and Garfinkle on primitive ideals, we are able to classify finite-dimensional irreducible modules for $U(\mathfrak{g}, e)$ and explicitly describe the component group action. These results are stated in Theorems 5.4 and 6.17 .

In Section 7, we use inductive methods to deduce Theorem 1.2. The important ingredients here are "Levi subalgebras" of $U(\mathfrak{g}, e)$ as defined in [Brown and Goodwin 2013a, §3] and changing highest weight theories. The latter is dealt with in [Brown and Goodwin 2013c] for the case of an even-multiplicity nilpotent orbit, and we observe here that there is an analogous theory in the present situation; see Proposition 4.6.

We note that if we were able to deal with the case where $\boldsymbol{p}$ has three parts by other means, for example from an explicit presentation of the finite $W$-algebras, then we would be able to remove the dependence on the results of Losev, Barbasch and Vogan, and Garfinkle. It would, therefore, be interesting and useful to have a presentation of such finite $W$-algebras.

\section{Overview of finite $W$-algebras}

2A. Definition of the finite $\boldsymbol{W}$-algebra $\boldsymbol{U}(\mathfrak{g}, \boldsymbol{e})$. Let $G$ be a reductive algebraic group over $\mathbb{C}$ with Lie algebra $\mathfrak{g}$. The finite $W$-algebra $U(\mathfrak{g}, e)$ is defined in terms of a nilpotent element $e \in \mathfrak{g}$. By the Jacobson-Morozov Theorem, $e$ embeds into an $\mathfrak{s l}_{2}$-triple $(e, h, f)$. The ad $h$ eigenspace decomposition gives a grading on $\mathfrak{g}$ :

$$
\mathfrak{g}=\bigoplus_{j \in \mathbb{Z}} \mathfrak{g}(j),
$$

where $\mathfrak{g}(j)=\{x \in \mathfrak{g} \mid[h, x]=j x\}$. Define the character $\chi: \mathfrak{g} \rightarrow \mathbb{C}$ by $\chi(x)=(x, e)$, where $(\cdot, \cdot)$ is a nondegenerate symmetric invariant bilinear form on $\mathfrak{g}$. Then we can define a nondegenerate symplectic form $\langle\cdot, \cdot\rangle$ on $\mathfrak{g}(-1)$ by $\langle x, y\rangle=\chi([y, x])$. Choose a Lagrangian subspace $\mathfrak{l} \subseteq \mathfrak{g}(-1)$ with respect to $\langle\cdot, \cdot\rangle$, and let $\mathfrak{m}=$ $\mathfrak{l} \oplus \bigoplus_{j \leq-2} \mathfrak{g}(j)$. Let $\mathfrak{m}_{\chi}=\{m-\chi(m) \mid m \in \mathfrak{m}\}$. The adjoint action of $\mathfrak{m}$ on $U(\mathfrak{g})$ leaves the left ideal $U(\mathfrak{g}) \mathfrak{m}_{\chi}$ invariant, so there is an induced adjoint action of $\mathfrak{m}$ on $Q_{\chi}=U(\mathfrak{g}) / U(\mathfrak{g}) \mathfrak{m}_{\chi}$. The space of fixed points $Q_{\chi}^{\mathfrak{m}}$ inherits a well-defined multiplication from $U(\mathfrak{g})$, making it an associative algebra, and we define the finite $W$-algebra to be

$$
U(\mathfrak{g}, e)=Q_{\chi}^{\mathfrak{m}}=\left\{u+U(\mathfrak{g}) \mathfrak{m}_{\chi} \in Q_{\chi} \mid[x, u] \in U(\mathfrak{g}) \mathfrak{m}_{\chi} \text { for all } x \in \mathfrak{m}\right\} .
$$

We also recall here that the centre $Z(\mathfrak{g})$ of $U(\mathfrak{g})$ maps into $U(\mathfrak{g}, e)$ via the inclusion $Z(\mathfrak{g}) \subseteq U(\mathfrak{g})$. Moreover, it is known that this defines an isomorphism between 
$Z(\mathfrak{g})$ and the centre of $U(\mathfrak{g}, e)$; see the footnote to [Premet 2007, Question 5.1]. We use this isomorphism to identify the centre of $U(\mathfrak{g}, e)$ with $Z(\mathfrak{g})$, which in particular allows us to define integral central characters for $U(\mathfrak{g}, e)$-modules.

Remark 2.2. There are different equivalent definitions of the finite $W$-algebra in the literature. Above we have given the Whittaker model definition, as it is the shortest and most convenient for our purposes here.

2B. Skryabin's equivalence and Losev's map of primitive ideals. The left $U(\mathfrak{g})$ module $Q_{\chi}$ is also a right $U(\mathfrak{g}, e)$-module, so there is a functor

$$
\mathscr{S}: U(\mathfrak{g}, e)-\bmod \rightarrow U(\mathfrak{g})-\bmod , \quad M \mapsto Q_{\chi} \otimes_{U(\mathfrak{g}, e)} M,
$$

where $M$ is a $U(\mathfrak{g}, e)$-module. Skryabin [2002] showed that $\mathscr{Y}$ is an equivalence of categories between $U(\mathfrak{g}, e)$-mod and the category of Whittaker modules for $e$, the category of $U(\mathfrak{g})$-modules on which $\mathfrak{m}_{\chi}$ acts locally nilpotently.

For an algebra $A$, let Prim $A$ denote the set of primitive ideals of $A$. Losev [2011a] showed that there exists a map

$$
.^{\dagger}: \operatorname{Prim} U(\mathfrak{g}, e) \rightarrow \operatorname{Prim} U(\mathfrak{g}), \quad I \mapsto I^{\dagger}
$$

with the following properties:

(1) It preserves central characters: $I \cap Z(\mathfrak{g})=I^{\dagger} \cap Z(\mathfrak{g})$ for any $I \in \operatorname{Prim}(U(\mathfrak{g}, e))$, under the identification of the centre of $U(\mathfrak{g}, e)$ with $Z(\mathfrak{g})$.

(2) It behaves well with respect to Skryabin's equivalence in the sense that

$$
\operatorname{Ann}_{U(\mathfrak{g})} \mathscr{Y}(M)=\left(\operatorname{Ann}_{U(\mathfrak{g}, e)} M\right)^{\dagger}
$$

for every irreducible $U(\mathfrak{g}, e)$-module $M$.

(3) Its restriction to $\operatorname{Prim}_{0} U(\mathfrak{g}, e)$, the set of primitive ideals of $U(\mathfrak{g}, e)$ of finite codimension, is a surjection onto $\operatorname{Prim}_{e} U(\mathfrak{g})$, the set of primitive ideals of $U(\mathfrak{g})$ with associated variety equal to $\overline{G \cdot e}$.

(4) Its fibres restricted to $\operatorname{Prim}_{0} U(\mathfrak{g}, e)$ are $C$-orbits, where $C$ is the component group of the centralizer of $e$. See, for example, the introduction to [Losev 2011a] for an explanation of the action of $C$ on $\operatorname{Prim}_{0} U(\mathfrak{g}, e)$.

2C. Highest weight theory and Losev's map. By using the highest weight theory for finite $W$-algebras developed by Brundan, Kleshchev and Goodwin in [Brundan et al. 2008] (abbreviated [BGK] in this section), the map ${ }^{\dagger}$ from the previous subsection can be explicitly calculated in terms of highest weight modules for $U(\mathfrak{g}, e)$ and $U(\mathfrak{g})$.

The key part of this highest weight theory is the use of a minimal Levi subalgebra $\mathfrak{g}_{0}$ that contains $e$. In [BGK, Theorem 4.3] it is proved that there is a certain 
subquotient of $U(\mathfrak{g}, e)$ that is isomorphic to $U\left(\mathfrak{g}_{0}, e\right)$. Then, in [BGK, §4.2], it is explained how a choice of a parabolic subalgebra $\mathfrak{q}$ with Levi factor $\mathfrak{g}_{0}$ leads to a highest weight theory for $U(\mathfrak{g}, e)$ in which $U\left(\mathfrak{g}_{0}, e\right)$ plays the role of the Cartan subalgebra in the usual highest weight theory for reductive Lie algebras. This leads to a definition of Verma modules for $U(\mathfrak{g}, e)$ by "parabolically inducing" $U\left(\mathfrak{g}_{0}, e\right)$ modules up to $U(\mathfrak{g}, e)$-modules. Then [BGK, Theorem 4.5] says that these Verma modules have irreducible heads, and that any finite-dimensional irreducible $U(\mathfrak{g}, e)$ module is isomorphic to one of these irreducible heads. This gives a method to explicitly parametrize finite-dimensional irreducible $U(\mathfrak{g}, e)$-modules, though a classification of $U\left(\mathfrak{g}_{0}, e\right)$-modules in general is unknown at present.

When $e$ is of standard Levi type, the classification of $U\left(\mathfrak{g}_{0}, e\right)$-modules is known. By a theorem of Kostant [1978] and the Harish-Chandra isomorphism, we have that $U\left(\mathfrak{g}_{0}, e\right) \cong Z\left(\mathfrak{g}_{0}\right) \cong S(\mathfrak{t})^{W_{0}}$, where $\mathfrak{t}$ is a maximal toral subalgebra of $\mathfrak{g}$ and $W_{0}$ is the Weyl group of $\mathfrak{g}_{0}$. Hence the finite-dimensional irreducible $U\left(\mathfrak{g}_{0}, e\right)$-modules are all one-dimensional, and they are parametrized by the $W_{0}$-orbits on $t^{*}$. We choose $\mathfrak{t}$ as specified in [BGK, §5.1], and let $\Lambda \in \mathfrak{t}^{*} / W_{0}$ be a $W_{0}$-orbit. In [BGK] an explicit isomorphism $U\left(\mathfrak{g}_{0}, e\right) \rightarrow S(\mathfrak{t})^{W_{0}}$ is given. Using this isomorphism and our choice of $\mathfrak{q}$, we let $M(\Lambda, \mathfrak{q})$ denote the Verma module for $U(\mathfrak{g}, e)$ induced from $\Lambda$, and we write $L(\Lambda, \mathfrak{q})$ for the irreducible head of $M(\Lambda, \mathfrak{q})$. We note that there are "shifts" involved in the isomorphisms above and thus in the definition of $M(\Lambda, \mathfrak{q})$ in [BGK, Sections 4 and 5].

Let $\mathfrak{u}$ be the nilradical of $\mathfrak{q}$ and let $\mathfrak{b}_{0}$ be a Borel subalgebra of $\mathfrak{g}_{0}$ containing $\mathfrak{t}$, so that $\mathfrak{b}=\mathfrak{b}_{0} \oplus \mathfrak{u}$ is a Borel subalgebra of $\mathfrak{g}$. For $\lambda \in \mathfrak{t}^{*}$, let $L(\lambda, \mathfrak{b})$ denote the highest weight irreducible $\mathfrak{g}$-module defined in terms of $\mathfrak{b}$, with highest weight $\lambda-\rho$ (where $\rho$ is the half-sum of the positive roots for $\mathfrak{b}$ ).

The theorem below allows us to explicitly calculate Losev's map $\cdot^{\dagger}$ on primitive ideals in terms of highest weight modules. In [BGK, §5.1] it was shown that this theorem follows from [Miličić and Soergel 1997, Theorem 5.1] and [BGK, Conjecture 5.3]. Also this last conjecture was verified in [Losev 2012, Theorem 5.1.1], except for a technical point which was resolved in [Brown and Goodwin 2013a, Proposition 3.10].

Theorem 2.3. Let $\Lambda \in \mathfrak{t}^{*} / W_{0}$ and let $\lambda \in \Lambda$ be antidominant for $\mathfrak{b}_{0}$. Then

$$
\left(\operatorname{Ann}_{U(\mathfrak{g}, e)} L(\Lambda, \mathfrak{q})\right)^{\dagger}=\operatorname{Ann}_{U(\mathfrak{g})} L(\lambda, \mathfrak{b}) .
$$

One consequence of this theorem is that if $e$ is not a special nilpotent element, then $U(\mathfrak{g}, e)$ has no finite-dimensional irreducible representations of integral central character. This is due to results of Barbasch and Vogan [1982; 1983], which imply that the associated variety of $A n_{U(\mathfrak{g})} L(\lambda, \mathfrak{b})$ is a special nilpotent orbit if and only if $\lambda$ is integral. 
The following theorem is Conjecture 5.2 of [BGK], which follows from Conjecture 5.3 of the same paper, as is explained in there.

Theorem 2.4. Let $\Lambda \in \mathfrak{t}^{*} / W_{0}$ and let $\lambda \in \Lambda$ be antidominant for $\mathfrak{b}_{0}$. Then $L(\Lambda, \mathfrak{q})$ is finite-dimensional if and only if the associated variety of $\operatorname{Ann}_{U(\mathfrak{g})} L(\lambda, \mathfrak{b})$ is equal to $\overline{G \cdot e}$.

\section{Combinatorics of s-tables and finite $W$-algebras}

3A. Realizations of $\mathfrak{s o}_{2 n+1}$ and $\mathfrak{s p}_{2 n}$. In the case $\mathfrak{g}=\mathfrak{s o}_{2 n+1}$, we realize $\mathfrak{g}$ in the following way: Let $V=\mathbb{C}^{2 n+1}$ have basis $\left\{e_{1}, \ldots, e_{n}, e_{0}, e_{-n}, \ldots, e_{-1}\right\}$. Then we take $\mathfrak{g l}_{2 n+1}=\operatorname{End}(V)$ as having basis $\left\{e_{i, j} \mid i, j=0, \pm 1, \ldots, \pm n\right\}$, where $e_{i, j} \in \operatorname{End}(V)$ is defined via $e_{i, j}\left(e_{k}\right)=\delta_{j, k} e_{i}$. We define the bilinear form $(\cdot, \cdot)$ on $V$ by declaring that $\left(e_{i}, e_{j}\right)=\delta_{i,-j}$. Then we set

$$
\mathfrak{g}=\mathfrak{s o}_{2 n+1}=\left\{x \in \mathfrak{g l}_{2 n+1} \mid(x v, w)=-(v, x w) \text { for all } v, w \in V\right\} .
$$

Note that $\mathfrak{g}$ has basis $\left\{f_{i, j} \mid i, j=0, \pm 1, \ldots, \pm n, i+j>0\right\}$, where $f_{i, j}=$ $e_{i, j}-e_{-j,-i}$. We choose $\mathfrak{t}=\left\{f_{i, i} \mid i=1, \ldots, n\right\}$ as a maximal toral subalgebra, so that $\mathrm{t}^{*}$ has basis $\left\{\epsilon_{i} \mid i=1, \ldots, n\right\}$, where $\epsilon_{i} \in \mathfrak{t}^{*}$ is defined via $\epsilon_{i}\left(f_{j, j}\right)=\delta_{i, j}$ for $i, j>0$. We write $\Phi$ for the root system of $\mathfrak{g}$ with respect to $\mathfrak{t}$. Let $\mathfrak{b}=\left\langle f_{i, j} \mid i \leq j\right\rangle$ be the Borel subalgebra of upper-triangular matrices in $\mathfrak{g}$. Then the corresponding system of positive roots is given by

$$
\Phi^{+}=\left\{\epsilon_{i} \pm \epsilon_{j} \mid 1 \leq i<j \leq n\right\} \cup\left\{\epsilon_{i} \mid i=1, \ldots, n\right\} .
$$

For $\mathfrak{g}=\mathfrak{s p}_{2 n}$, we let $V=\mathbb{C}^{2 n}$ have basis $\left\{e_{1}, \ldots, e_{n}, e_{-n}, \ldots, e_{-1}\right\}$. Then we realize $\mathfrak{g l}_{2 n}=\operatorname{End}(V)$ as having basis $\left\{e_{i, j} \mid i, j= \pm 1, \ldots, \pm n\right\}$, where $e_{i, j} \in$ $\operatorname{End}(V)$ is defined via $e_{i, j}\left(e_{k}\right)=\delta_{j, k} e_{i}$. We define the bilinear form $(\cdot, \cdot)$ on $V$ by declaring that $\left(e_{i}, e_{j}\right)=\operatorname{sign}(i) \delta_{i,-j}$, and set

$$
\mathfrak{g}=\mathfrak{s p}_{2 n}=\left\{x \in \mathfrak{g l}_{2 n} \mid(x v, w)=-(v, x w) \text { for all } v, w \in V\right\} .
$$

Then $\mathfrak{g}$ has basis $\left\{f_{i, j} \mid i, j= \pm 1, \ldots, \pm n, i+j \geq 0\right\}$, where $f_{i, j}=e_{i, j}-$ $\operatorname{sign}(i) \operatorname{sign}(j) e_{-j,-i}$. We choose $\mathfrak{t}=\left\{f_{i, i} \mid i=1, \ldots, n\right\}$ as a maximal toral subalgebra, so that $\mathfrak{t}^{*}$ has basis $\left\{\epsilon_{i} \mid i=1, \ldots, n\right\}$, where $\epsilon_{i} \in \mathfrak{t}^{*}$ is defined via $\epsilon_{i}\left(f_{j, j}\right)=\delta_{i, j}$ for $i, j>0$. We write $\Phi$ for the root system of $\mathfrak{g}$ with respect to $\mathfrak{t}$. We choose the Borel subalgebra $\mathfrak{b}=\left\langle f_{i, j} \mid i \leq j\right\rangle$ of upper-triangular matrices in $\mathfrak{g}$. Then the corresponding system of positive roots is given by

$$
\Phi^{+}=\left\{\epsilon_{i} \pm \epsilon_{j} \mid 1 \leq i<j \leq n\right\} \cup\left\{2 \epsilon_{i} \mid i=1, \ldots, n\right\} .
$$

3B. Standard Levi nilpotent elements and symmetric pyramids. Recall from the introduction that we are considering nilpotent orbits in $\mathfrak{g}$ that are special and standard 
Levi, but not even-multiplicity. The Jordan type for such a nilpotent orbit is of the form

$$
\boldsymbol{p}=\left(p_{1}^{2} \leq \cdots \leq p_{d-1}^{2}<p_{0} \leq p_{d}^{2} \leq \cdots \leq p_{r}^{2}\right) .
$$

Moreover, $p_{i}$ must be odd for all $i \geq d$ when $\mathfrak{g}=\mathfrak{s o}_{2 n+1}$ or $p_{i}$ must be even for all $i<d$ when $\mathfrak{g}=\mathfrak{s p}_{2 n}$. As explained in the introduction, the condition that $\boldsymbol{p}$ has only one part of odd multiplicity is due to the standard Levi assumption, and the parity conditions are due to the assumption that the corresponding orbit is special.

Also recall from the introduction the definition of the symmetric pyramid $P$ for $\boldsymbol{p}$. We form a diagram $K$ called the coordinate pyramid for $\boldsymbol{p}$ by filling the boxes of $P$ with $1, \ldots, n,-n, \ldots,-1$ if $\mathfrak{g}=\mathfrak{s p}_{2 n}$ or with $1, \ldots, n, 0,-n, \ldots,-1$ if $\mathfrak{g}=\mathfrak{s o}_{2 n}$, across rows from top to bottom. For example, for $\mathfrak{g}=\mathfrak{s p}_{18}$ and $\boldsymbol{p}=\left(2^{2}, 4,5^{2}\right)$, we have

$$
K=\begin{array}{|l|l|l|l|l|l|}
\cline { 2 - 3 } \multicolumn{1}{c|}{} & 1 & \multicolumn{2}{|c|}{} & \multicolumn{2}{c}{} \\
\hline 3 & 4 & 5 & 6 & 7 \\
\hline & 8 & 9 & -9 & -8 & \\
\hline-7 & -6 & -5 & -4 & -3 \\
\hline \multicolumn{7}{c}{} & -2 & -1 &
\end{array}
$$

We let $\operatorname{col}(i)$ denote the $x$-coordinate of the centre of the box of $K$ that contains $i$. However, we use row $(i)$ to denote the row of $K$ that contains $i$ when we label the rows of $K$ by $1, \ldots, r, 0,-r, \ldots,-1$ from top to bottom, so that $p_{i}$ is the length of row $i$.

We define $e \in \mathfrak{g}$ by

$$
e=\sum_{i, j} f_{i, j}
$$

where the sum is over all adjacent pairs $i \quad j$ in $K$, so that $e$ is in the nilpotent $G$-orbit with Jordan type $\boldsymbol{p}$.

We also use $K$ to conveniently define many of the objects required for the definition of $U(\mathfrak{g}, e)$ and the highest weight theory.

Let $h=\sum_{i=1}^{n}-\operatorname{col}(i) f_{i, i}$; then $(e, h, f)$ is an $\mathfrak{s l}_{2}$-triple for some $f \in \mathfrak{g}$. Furthermore, the grading from $(2.1)$ on $\mathfrak{g}$ is given by $\mathfrak{g}(k)=\left\langle f_{i, j} \mid \operatorname{col}(j)-\operatorname{col}(i)=k\right\rangle$. For our Lagrangian subspace of $\mathfrak{g}(-1)$, we let

$$
\mathfrak{l}=\left\langle f_{i, j} \mid \operatorname{col}(i)-\operatorname{col}(j)=1, \operatorname{row}(i)<\operatorname{row}(j)\right\rangle .
$$

Then we have that $\mathfrak{m}=\mathfrak{l} \oplus\left\langle f_{i, j} \mid \operatorname{col}(i)-\operatorname{col}(j)>1\right\rangle$, and we use these choices of $e$ and $\mathfrak{m}$ to form the finite $W$-algebra $U(\mathfrak{g}, e)$ as in Section 2A. 
We take $\mathfrak{g}_{0}=\left\langle f_{i, j} \mid \operatorname{row}(i)=\operatorname{row}(j)\right\rangle$. So $\mathfrak{g}_{0}$ is a minimal Levi subalgebra which contains $e$, and $e$ is a regular nilpotent element of $\mathfrak{g}_{0}$. In the case $\mathfrak{g}=\mathfrak{s o}_{2 n+1}$, we have

$$
\mathfrak{g}_{0} \cong \mathfrak{s o}_{p_{0}} \oplus \bigoplus_{i=1}^{r} \mathfrak{g l}_{p_{i}}
$$

and in the case $\mathfrak{g}=\mathfrak{s p}_{2 n}$ we have

$$
\mathfrak{g}_{0} \cong \mathfrak{s p}_{p_{0}} \oplus \bigoplus_{i=1}^{r} \mathfrak{g l}_{p_{i}} .
$$

We choose $\mathfrak{q}=\left\langle f_{i, j}\right|$ the row containing $i$ is above or equal to the row containing $\left.j\right\rangle$. Then $\mathfrak{q}$ is a parabolic subalgebra of $\mathfrak{g}$ with Levi factor $\mathfrak{g}_{0}$. Let $\mathfrak{b}_{0}=\mathfrak{b} \cap \mathfrak{g}_{0}$, so that $\mathfrak{b}_{0}$ is a Borel subalgebra of $\mathfrak{g}_{0}$ that satisfies $\mathfrak{b}=\mathfrak{b}_{0} \oplus \mathfrak{u}$, where $\mathfrak{u}$ is the nilradical of $\mathfrak{q}$.

3C. Tables and s-tables. We use the definitions and notation regarding frames, tables, s-frames and s-tables from [Brown and Goodwin 2013a, §4]. Below we explain how these are used to label highest weight modules for $U(\mathfrak{g}, e)$.

For this purpose, we let $W_{r}$ be the Weyl group of type $\mathrm{B}_{r}$, which acts on $\{0, \pm 1, \ldots, \pm r\}$ in the natural way. We denote the standard generators of $W_{r}$ by $\bar{s}_{i}=(i, i+1)(-i,-i-1)$, for $i=1, \ldots, r-1$. Let $\bar{S}_{r}$ be the subgroup of $W_{r}$ generated by $\bar{s}_{i}$ for $i=1, \ldots, r-1$.

Given $\sigma \in W_{r}$, we define $\sigma \cdot P$ to be the diagram obtained from $P$ by permuting rows according to $\sigma$, so that $\sigma \cdot P$ is an $s$-frame (recall that an $s$-frame is a collection of connected rows of boxes in the plane arranged symmetrically around the origin). We recall that by an s-table with frame $\sigma \cdot P$, we mean a skew-symmetric (with respect to the origin) filling of $\sigma \cdot P$ with complex numbers. Then we define $\operatorname{sTab}(\sigma \cdot P)$ to be the set of s-tables with frame $\sigma \cdot P$ such that all entries are integers if $\mathfrak{g}=\mathfrak{s p}_{2 n}$, and either all entries are in $\mathbb{Z}$ or all entries are in $\frac{1}{2}+\mathbb{Z}$ if $\mathfrak{g}=\mathfrak{s o}_{2 n+1}$.

We let $\sigma \cdot K$ be the s-table obtained from $K$ by permuting rows according to $\sigma$. Now given $A \in \operatorname{sTab}(\sigma \cdot P)$, we define $\lambda_{A}=\sum_{i=1}^{n} a_{i} \epsilon_{i}$, where $a_{i}$ is the entry of $A$ in the same box as $i$ in $\sigma \cdot K$. In this way we get an identification of $\operatorname{sTab}(\sigma \cdot P)$ with the set of integral weights in $t^{*}$; we write $t_{\mathbb{Z}}^{*}$ for the set of integral weights of $t$.

The row equivalence class of an s-table is the set of s-tables that can be created by permuting entries within rows. We let $\operatorname{sRow}(\sigma \cdot P)$ denote the set of row equivalence classes of $\operatorname{sTab}(\sigma \cdot P)$. Then $\operatorname{sRow}(\sigma \cdot P)$ is identified naturally with $\mathfrak{t}_{\mathbb{Z}}^{*} / W_{0}$, where $W_{0}$ is the Weyl group of $\mathfrak{g}_{0}$. Let $\operatorname{sTab}^{\leq}(\sigma \cdot P)$ denote the elements of $\operatorname{sTab}(\sigma \cdot P)$ that have nondecreasing rows. Then every element of that $\operatorname{sRow}(\sigma \cdot P)$ contains a unique element of $\operatorname{sTab}^{\leq}(\sigma \cdot P)$.

We label the rows of $\sigma \cdot K$ with $1, \ldots, r, 0,-r, \ldots,-1$ from top to bottom. Now, we define $\mathfrak{q}_{\sigma}$ to be generated by the by $f_{i j}$ for which the row of $\sigma \cdot K$ in 
which $i$ appears is above or equal to the row containing $j$. Then $\mathfrak{q}_{\sigma}$ is a parabolic subalgebra of $\mathfrak{g}$ with Levi factor $\mathfrak{g}_{0}$, so we can use it to define the irreducible highest weight modules $L\left(\Lambda, \mathfrak{q}_{\sigma}\right)$, for $\Lambda \in \mathfrak{t}^{*} / W_{0}$ as defined in Section $2 \mathrm{C}$.

Given $\Lambda \in \mathfrak{t}_{\mathbb{Z}}^{*} / W_{0}$, there is a unique $A \in \operatorname{sTab}^{\leq}(\sigma \cdot P)$ whose row equivalence class $\bar{A} \in \operatorname{sRow}(P)$ is identified with $\Lambda$ as above. We let $L_{\sigma}(A)$ denote $L\left(\Lambda, \mathfrak{q}_{\sigma}\right)$.

Let $\mathfrak{u}_{\sigma}$ be the nilradical of $\mathfrak{q}_{\sigma}$, and define $\mathfrak{b}_{\sigma}=\mathfrak{b}_{0} \oplus \mathfrak{u}_{\sigma}$, which is a Borel subalgebra of $\mathfrak{g}$. We write $L_{\sigma}\left(\lambda_{A}\right)$ for the irreducible highest weight $U(\mathfrak{g})$-module with respect to $\mathfrak{b}_{\sigma}$, with highest weight $\lambda_{A}-\rho_{\sigma}$, where $\rho_{\sigma}$ is the half-sum of positive roots for $\mathfrak{b}_{\sigma}$.

Now Theorem 2.3 can be restated in our present notation as follows:

Theorem 3.3. Let $\sigma \in W_{r}$ and $A \in \operatorname{sTab}^{\leq}(\sigma \cdot P)$. Then $\left(\operatorname{Ann}_{U(\mathfrak{g}, e)} L_{\sigma}(A)\right)^{\dagger}=$ $\operatorname{Ann}_{U(\mathfrak{g})} L_{\sigma}\left(\lambda_{A}\right)$.

We are mainly interested in the case where $\sigma=1$. Here we have $\mathfrak{q}_{\sigma}=\mathfrak{q}$, and we write $L(A)$ instead of $L_{1}(A)$ and $L\left(\lambda_{A}\right)$ instead of $L_{1}\left(\lambda_{A}\right)$ for $A \in \operatorname{sTab}(P)$.

Thanks to Theorem 3.3, our goal of classifying the finite-dimensional irreducible $U(\mathfrak{g}, e)$-modules and understanding the component group action on these modules can be broken down to answering the following questions:

(1) For which $A \in \operatorname{sTab}^{\leq}(P)$ is the associated variety of $\operatorname{Ann}_{U(\mathfrak{g})} L\left(\lambda_{A}\right)$ equal to $\overline{G \cdot e}$ ?

(2) Given $A \in \operatorname{sTab}^{\leq}(P)$ such that $L(A)$ is finite-dimensional, which $B \in \mathrm{sTab}^{\leq}(P)$ satisfy $\operatorname{Ann}_{U(\mathfrak{g})} L\left(\lambda_{A}\right)=\operatorname{Ann}_{U(\mathfrak{g})} L\left(\lambda_{B}\right)$ ?

In the case that $\boldsymbol{p}$ has three parts, we answer these two questions in Sections 5 and 6. The key ingredients in answering the first question are the Robinson-Schensted and Barbasch-Vogan algorithms explained in Section 4A and Section 4C. For the second question, we use Vogan's $\tau$-equivalence on integral weights of $\mathfrak{g}$, which is explained in Section 4D.

In moving from the three row case to the general case, a key role is played by the different choices of highest weight theories determined by the different parabolic subalgebras $\mathfrak{q}_{\sigma}$ for $\sigma \in W_{r}$. This dependence follows easily from the results for the case of even-multiplicity nilpotent elements established in [Brown and Goodwin 2013c], which hold in the present situation; the key result for us is Proposition 4.6. We also require the explicit description of the action of the component group on the set of finite-dimensional irreducible $U(\mathfrak{g}, e)$-modules in terms of s-tables, which is given in Proposition 7.1. The proof of Theorem 1.2 for the general case is then dealt with in Section 7B.

3D. The component group. Recall that $C$ denotes the component group of the centralizer of $e$ in $G$. Here we take $G$ to be the adjoint group of $\mathfrak{g}$, so $G$ is either $\mathrm{SO}_{2 n+1}$ or $\mathrm{PSp}_{2 n}$. 
A specific realization of $C$ is given as follows: Let $0<p_{i_{1}}<\cdots<p_{i_{s}}$ be the maximal distinct parts of $\boldsymbol{p}$ such that $p_{i_{j}} \neq p_{0}$ and $p_{i_{j}}$ is odd (respectively even) when $\mathfrak{g}=\mathfrak{s o}_{2 n+1}$ (respectively $\left.\mathfrak{s p}_{2 n}\right)$; by maximal, we mean that if $p_{k}=p_{i_{j}}$, then $k \leq i_{j}$. Define the matrices $c_{1}, \ldots, c_{s}$ corresponding to $p_{i_{1}}, \ldots, p_{i_{s}}$ for $p_{i_{k}} \neq p_{0}$ by setting

$$
c_{k}=\sum_{\begin{array}{c}
-n \leq i, j \leq n \\
\operatorname{col}(i) \operatorname{col}(j) \\
\operatorname{row}(i)=i_{k} \\
\operatorname{row}(j)=-i_{k}
\end{array}} \operatorname{sign}(\operatorname{col}(i))\left(e_{i, j}+e_{j, i}\right)+\sum_{\begin{array}{r}
-n \leq i \leq n \\
\operatorname{row}(i) \neq \pm i_{k}
\end{array}} e_{i, i} .
$$

Then one can calculate that $c_{k}$ centralizes $e$. Furthermore, the argument used in [Brown 2011, Section 6] can be adapted to show that the images of $c_{1}, \ldots, c_{s}$ in $C$ generate $C \cong \mathbb{Z}_{2}^{s}$.

As mentioned in Section 2B, there is an action of $C$ on Prim $U(\mathfrak{g}, e)$, and thus on isomorphism classes of irreducible modules, and, as explained in [Brown and Goodwin 2013a, §2.3], this can be seen as "twisting" modules by elements of $C$ (up to isomorphism). Given an irreducible $U(\mathfrak{g}, e)$-module $L$ and $b \in C$, we write $b \cdot L$ for the twisted module; we note that this is a minor abuse of notation as $b \cdot L$ is only defined up to isomorphism.

\section{Some combinatorics for s-tables}

4A. The Robinson-Schensted algorithm. We use the formulation of the Robinson-Schensted algorithm from [Brown and Goodwin 2013a, §4]. We denote the Robinson-Schensted algorithm by RS and recall that it takes as input a word of integers (or more generally complex numbers) or a table and outputs a tableau.

There are two lemmas about the Robinson-Schensted algorithm that we use repeatedly in the sequel. We state them below for convenience; they can be found in [Fulton 1997, §3]. For a word $w$, we define $\ell(w, k)$ to be the maximum possible sum of the lengths of $k$ disjoint weakly increasing subsequences of $w$, and $6(w, k)$ to be the maximum possible sum of the lengths of $k$ disjoint strictly decreasing subsequences of $w$. We write $\operatorname{part}(T)$ to denote the partition underlying a tableau $T$.

Lemma 4.1. Let $w$ be a word of integers and let $\boldsymbol{q}=\left(q_{1} \geq \cdots \geq q_{n}\right)=\operatorname{part}(\operatorname{RS}(w))$. Then for all $k \geq 1, \ell(w, k)=q_{1}+\cdots+q_{k}$.

Lemma 4.2. Let $w$ be a word of integers and let $\boldsymbol{q}^{T}=\left(q_{1}^{*} \geq \cdots \geq q_{n}^{*}\right)$ be the dual partition to $\boldsymbol{q}=\operatorname{part}(\mathrm{RS}(w))$. Then for all $k \geq 1,6(w, k)=q_{1}^{*}+\cdots+q_{k}^{*}$.

An elementary fact about the Robinson-Schensted algorithm, required later, is stated in Lemma 4.3 below; it is easily deduced from Lemma 4.1. Suppose $u, w$ are words of integers and $a, b$ are integers such that $a>b$; then we say the transposition 
of the word uabw to ubaw is a larger-smaller transposition. Also, we refer the reader to [Fulton 1997, §2] for the definition of Knuth equivalences.

Lemma 4.3. If $u$ and $w$ are words of integers and $w$ can be obtained from $u$ by a sequence of Knuth equivalences and larger-smaller transpositions, then $\operatorname{part}(\operatorname{RS}(u)) \leq \operatorname{part}(\operatorname{RS}(w))$.

The following theorem extends Theorem 4.6 of [Brown and Goodwin 2013a] and is important for us later. In the statement, $P$ is the symmetric pyramid for the partition $\boldsymbol{p}$, as in the previous section. Also, recall we defined the notion of a justified row-equivalent to column-strict s-table in the introduction.

Theorem 4.4. Let $A, B \in \mathrm{sTab}^{\leq}(P)$. Then:

(i) $A$ is justified row-equivalent to column-strict if and only if $\operatorname{part}(\operatorname{RS}(A))=\boldsymbol{p}$.

(ii) If $\operatorname{part}(\mathrm{RS}(A))=\boldsymbol{p}$, then $\mathrm{RS}(A)=\mathrm{RS}(B)$ if and only if $A=B$.

Proof. Part (i) can be proved in the same way as [Brown and Goodwin 2013a, Theorem 4.6]. We just need to check the proof still holds if $A$ has an odd number of rows and the middle row of $A$ is not $A$ 's longest row. The only thing to check is that there is a sequence of row swaps that transforms $A$ into a tableau such that the convexity conditions required by Lemma 4.9 of the same reference are satisfied, which is clear.

To prove (ii), we simply note that each row swap from the sequence of row swaps from (i) which turns $A$ into a tableau is invertible.

Lastly in this section we give the following theorem, which is important later on:

Theorem 4.5. Let $A, B \in \operatorname{sTab}^{c}(P)$. Suppose that $\operatorname{Ann}_{U(\mathfrak{g})} L\left(\lambda_{A}\right)=\operatorname{Ann}_{U(\mathfrak{g})} L\left(\lambda_{B}\right)$. Then $A=B$.

Proof. First, we need to briefly explain some of the results of Garfinkle [1990; 1993]. Section 2 of [Garfinkle 1990] defines the map $L: W_{n} \rightarrow \operatorname{Dom}_{n}$, where $\operatorname{Dom}_{n}$ denotes the set of domino tableaux for $W_{n}$ (see the appendix to this paper for more information on domino tableaux). Section 5 of the same work defines the map $S: \operatorname{Dom}_{n} \rightarrow \operatorname{sDom}_{n}$, where $\operatorname{sDom}_{n}$ denotes the set of domino tableaux for $W_{n}$ of special shape (a domino tableau has special shape if its underlying partition is the Jordan type of a special nilpotent element of $\mathfrak{g}$ ). Furthermore, $S$ restricted to sDom is the identity map.

For $\lambda \in \mathfrak{t}^{*}$, let $\operatorname{Prim}_{\lambda} U(\mathfrak{g})$ denote the primitive ideals of $U(\mathfrak{g})$ of central character $\lambda$. Suppose $\lambda \in \mathfrak{t}^{*}$ is antidominant and integral. Now, Theorem 3.5.11 of [Garfinkle 1993] states that the map cl : $\operatorname{Prim}_{\lambda} U(\mathfrak{g}) \rightarrow \operatorname{sDom}(n)$ given by $\operatorname{cl}($ Ann $L(w \lambda))=S(L(w))$ is a bijection.

Next we need to know that Garfinkle's map $L$ gives the same result as the Robinson-Schensted algorithm. This is provided in the appendix by Proposition A.4, 
which is simply a rephrasing of [van Leeuwen 1996, Proposition 4.2.3]. More specifically, the Robinson-Schensted algorithm outputs a tableau. There is a canonical way to associate a tableau that has been outputted by the Robinson-Schensted algorithm with a domino tableau (namely the algorithm DT from the appendix). Now, Proposition A.4 says that if we identify the output of the Robinson-Schensted algorithm with a domino tableau, then the result is the same as we would get from Garfinkle's $L$ algorithm.

Now, to prove the theorem, note that since $A$ and $B$ are justified row-equivalent to column-strict, by Theorem 4.4, $\operatorname{part}(\operatorname{RS}(A))=\operatorname{part}(\operatorname{RS}(B))=\boldsymbol{p}$. Since

$$
\operatorname{Ann}_{U(\mathfrak{g})} L\left(\lambda_{A}\right)=\operatorname{Ann}_{U(\mathfrak{g})} L\left(\lambda_{B}\right),
$$

the above discussion allows us to deduce that $\operatorname{RS}(A)=\mathrm{RS}(B)$. Now, the theorem follows from Theorem 4.4.

4B. Row swapping. In the proof of Theorem 4.4 above, we have mentioned the row swapping operations $s_{i} \star$ on tables, as defined in [Brown and Goodwin 2013a, $\S 4 ; 2013 \mathrm{c}, \S 4]$. An important ingredient for the definition of these row swapping operations is the notion of best fitting as defined in [Brown and Goodwin 2013a, $\S 4]$, which we use repeatedly in the following.

We also require the operations $\bar{s}_{i} \star$ for s-tables, and we use the notation from [Brown and Goodwin 2013c, §5]. Recall that for $\sigma \in W_{r}$ and an s-table $A \in$ $\operatorname{sTab}^{\leq}(\sigma \cdot P)$, either $\bar{s}_{i} \star A$ is undefined or it is an element of $\operatorname{sTab}^{\leq}\left(\bar{s}_{i} \sigma \cdot P\right)$. These operations can be extended to operations by elements of $\bar{S}_{r}$; the proof of Proposition 5.5(i) of the same reference goes through in our situation to show that this is well defined.

The following proposition is a version of [Brown and Goodwin 2013c, Proposition 5.3(ii)] in the present setting, and its proof adapts immediately:

Proposition 4.6. Let $\sigma \in W_{r}, \tau \in \bar{S}_{r}$ and $A \in \mathrm{sTab}^{\leq}(\sigma \cdot P)$. Suppose that $\tau \star A$ is defined. Then $L_{\sigma}(A) \cong L_{\tau \sigma}(\tau \star A)$.

Also, we state the following lemma, as it is key in the proof of Theorem 1.2. It is [Brown and Goodwin 2013a, Lemma 5.11], adapted to our situation, and the same proof holds. In the statement, $A_{r}^{1}$ denotes the table formed by rows 1 to $r$ of $A$.

Lemma 4.7. For $A \in \mathrm{sTab}^{\leq}(P)$, suppose that $L(A)$ is finite-dimensional, and let $\tau \in \bar{S}_{r}$. Then $A_{r}^{1}$ is justified row-equivalent to column-strict and $\tau \star A$ is defined.

4C. The Barbasch-Vogan algorithm. The Barbasch-Vogan algorithm [1982] takes as input $\lambda$, an integral weight for a classical Lie algebra of type B or C, and outputs $\mathrm{BV}(\lambda)$, the Jordan type of the associated variety of $\mathrm{Ann}_{U(\mathfrak{g})} L(\lambda)$. Below we recall the description of it given in [Brown and Goodwin 2013a, §5.2]. We note that there is a version of it for type $\mathrm{D}$, but we do not require that here. 
We need to define the content of a partition. Let $\boldsymbol{q}=\left(q_{1} \leq q_{2} \leq \cdots \leq q_{m}\right)$ be a partition. By inserting 0 at the beginning if necessary, we may assume that $m$ is odd. Let $\left(s_{1}, \ldots, s_{k}\right),\left(t_{1}, \ldots, t_{l}\right)$ be such that

$$
\left\{q_{1}, q_{2}+1, q_{3}+2, \ldots, q_{r}+r-1\right\}=\left\{2 s_{1}, \ldots, 2 s_{k}, 2 t_{1}+1, \ldots, 2 t_{l}+1\right\}
$$

(as unordered lists). Now, we define the content of $\boldsymbol{q}$ to be the unordered list

$$
\operatorname{content}(\boldsymbol{q})=\left\{s_{1}, \ldots, s_{k}, t_{1}, \ldots, t_{l}\right\} .
$$

\section{Algorithm:}

Input: $\lambda=\sum_{i=1}^{n} a_{i} \epsilon_{i}$ an integral weight in $\mathfrak{t}^{*}$.

Step 1: Calculate $\boldsymbol{q}=\operatorname{part}\left(\operatorname{RS}\left(a_{1}, \ldots, a_{n},-a_{n}, \ldots,-a_{1}\right)\right)$.

Step 2: Calculate content $(\boldsymbol{q})$.

Let $\left(u_{1} \leq \cdots \leq u_{2 k+1}\right)$ be the sorted list with the same entries as $\operatorname{content}(\boldsymbol{q})$.

For $i=1, \ldots, k+1$, let $s_{i}=u_{2 i-1}$.

For $i=1, \ldots, k$, let $t_{i}=u_{2 i}$.

Step 3: Form the list $\left(2 s_{1}+1, \ldots, 2 s_{k+1}+1,2 t_{1}, \ldots, 2 t_{k}\right)$.

In either case, let $\left(v_{1}<\cdots<v_{k}\right)$ be this list after sorting.

Output: $\operatorname{BV}(\lambda)=\boldsymbol{q}^{\prime}=\left(v_{1}, v_{2}-1, \ldots, v_{2 k+1}-2 k\right)$.

We note that the output partition $\boldsymbol{q}^{\prime}$ (potentially with an extraneous zero at the beginning) is the Jordan type of a special nilpotent orbit of $\mathfrak{g}$; this was proved in [Barbasch and Vogan 1982].

For our purposes in this paper, we also need a modified version of the algorithm to use in the case $\mathfrak{g}=\mathfrak{s o}_{2 n+1}$. This modified version is denoted by $\mathrm{BV}^{\prime}$. It works in exactly the same way as BV, except that in Step 1 instead of calculating $\operatorname{RS}\left(a_{1}, \ldots, a_{n},-a_{n}, \ldots,-a_{1}\right)$ we calculate $\operatorname{RS}\left(a_{1}, \ldots, a_{n}, 0,-a_{n}, \ldots,-a_{1}\right)$.

In Corollary A.7, in the appendix to this paper, it is proved that

$$
\mathrm{BV}(\lambda)=\mathrm{BV}^{\prime}(\lambda)
$$

for $\lambda \in \mathfrak{t}^{*}$ in the case $\mathfrak{g}=\mathfrak{s o}_{2 n+1}$. This proof of this is entirely combinatorial and may be of independent interest so it is has been placed in an appendix. In light of this, we redefine $\operatorname{BV}(\lambda)$, so that it is the old $\mathrm{BV}(\lambda)$ in the case $\mathfrak{g}=\mathfrak{s p}_{2 n}$ and is $\mathrm{BV}^{\prime}(\lambda)$ in the case $\mathfrak{g}=\mathfrak{s o}_{2 n+1}$.

For convenience of reference later in this paper we state the following theorem from [Barbasch and Vogan 1982]:

Theorem 4.8. Let $\lambda \in \mathfrak{t}_{\mathbb{Z}}^{*}$. Then the associated variety to $\mathrm{Ann}_{U(\mathfrak{g})} L(\lambda)$ is equal to the nilpotent $G$-orbit with Jordan type given by $\mathrm{BV}(\lambda)$. 
4D. The $\tau$-equivalence. The Barbasch-Vogan algorithm is used to find the associated variety of $\operatorname{Ann}_{U(\mathfrak{g})}(L(\lambda))$; however, in order to determine the action of the component group we need to be able to determine when $\operatorname{Ann}_{U(\mathfrak{g})} L(\mu)=\operatorname{Ann}_{U(\mathfrak{g})} L(\lambda)$. This can be done using the $\tau$-equivalence. This is an equivalence relation on the set of integral weights of $t$.

Recall our realization of $\mathfrak{g}$ and its Borel subalgebra $\mathfrak{b}$ defined in Section 3A, and recall that $\Phi^{+}$is the system of positive roots for $\mathfrak{g}$ defined from $\mathfrak{b}$ and $\mathfrak{t}$. Let $\Delta$ be the base of $\Phi$ corresponding to $\Phi^{+}$. Also, for $\alpha \in \Phi$, let $s_{\alpha} \in W$ denote the corresponding reflection in the Weyl group $W$ of $\mathfrak{g}$ with respect to $t$. For $w \in W$, let

$$
S(w)=\left\{\alpha \in \Phi^{+} \mid w \alpha \notin \Phi^{+}\right\} .
$$

Now let

$$
\tau(w)=S(w) \cap \Delta .
$$

Suppose that $\lambda \in \mathfrak{t}^{*}$ is an integral antidominant weight. Let $\alpha \in \Delta$ and $w \in W$. Suppose that $\alpha \in \tau\left(w^{-1}\right)$ satisfies $\tau\left(w^{-1} s_{\alpha}\right) \nsubseteq \tau\left(w^{-1}\right)$. Then

$$
\operatorname{Ann}_{U(\mathfrak{g})} L\left(s_{\alpha} w \lambda\right)=\operatorname{Ann}_{U(\mathfrak{g})} L(w \lambda)
$$

by [Joseph 1977, Theorem 5.1]; see also [Barbasch and Vogan 1982, Proposition 15]. With this in mind, we define the $\tau$-equivalence on integral weights to be the equivalence relation generated by declaring that

$$
\lambda_{1} \sim^{\tau} \lambda_{2}
$$

if there exist an antidominant integral weight $\lambda^{\prime}$, and elements $w \in W$ and $\alpha \in \Delta$ such that $\lambda_{1}=w \lambda^{\prime}, \lambda_{2}=s_{\alpha} w \lambda^{\prime}$ and $\tau\left(w^{-1} s_{\alpha}\right) \nsubseteq \tau\left(w^{-1}\right)$. In fact, the next theorem states that the $\tau$-equivalence is a complete invariant on primitive ideals:

Theorem 4.9 [Garfinkle 1993, Theorem 3.5.9]. Let $\lambda, \mu \in \mathfrak{t}^{*}$ be integral weights. Then $\lambda \sim^{\tau} \mu$ if and only if $\operatorname{Ann}_{U(\mathfrak{g})} L(\lambda)=\operatorname{Ann}_{U(\mathfrak{g})} L(\mu)$.

We identify the weight $\sum_{i=1}^{n} a_{i} \epsilon_{i} \in \mathfrak{t}^{*}$ with the list $\left(a_{1}, \ldots, a_{n}\right)$. Then one can check that the $\tau$-equivalence is generated by the following three relations:

(R1) $\left(a_{1}, \ldots, a_{n}\right) \sim^{\tau}\left(b_{1}, \ldots, b_{n}\right)$ if $\left(a_{1}, \ldots, a_{n}\right) \sim^{K}\left(b_{1}, \ldots, b_{n}\right)$;

(R2) $\left(a_{1}, \ldots, a_{n}\right) \sim^{\tau}\left(a_{1}, \ldots, a_{n-1},-a_{n}\right)$ if $\left|a_{n-1}\right|<\left|a_{n}\right|$;

(R3) $\left(a_{1}, \ldots, a_{n}\right) \sim^{\tau}\left(a_{1}, \ldots, a_{n-2}, a_{n}, a_{n-1}\right)$ if $a_{n-1} a_{n}<0$.

In (R1), $\sim^{K}$ denotes Knuth equivalence, as defined in [Fulton 1997, §2].

The references for the results in this section often only deal with the case of regular weights. However, [Jantzen 1983, Lemma 5.6] implies that they are valid for nonregular weights too. 


\section{The three row case for $\mathfrak{g}=\mathfrak{s p}_{2 n}$}

Let $\mathfrak{g}=\mathfrak{s p}_{2 n}$ and suppose that $\boldsymbol{p}$ has three parts. Then we write $\boldsymbol{p}=\left(l^{2}, m\right)$, where $l$ must be even if $l<m$. In this section we classify the finite-dimensional $U(\mathfrak{g}, e)$ modules, and we use the $\tau$-equivalence to describe the component group action on these modules.

Let $C$ be the component group of $e$, so

$$
C= \begin{cases}\langle c\rangle \cong \mathbb{Z}_{2} & \text { if } l \text { is even and } l \neq m, \\ 1 & \text { otherwise. }\end{cases}
$$

The lemma below deals with the (easy) cases where $l$ is even and $l \leq m$, or $l$ is odd (in which case $l>m$ ):

Lemma 5.1. Suppose that $A \in \mathrm{sTab}^{\leq}(P)$ and $l$ is even and $l \leq m$, or $l$ is odd. Then $L(A)$ is finite-dimensional if and only if $A$ is justified row-equivalent to columnstrict. Furthermore, in the case that $l$ is even and $l<m$, if $L(A)$ is finite-dimensional, then $c \cdot L(A) \cong L(A)$.

Proof. First, we consider the case where $l$ is even and $l \leq m$. So content $(l, l, m)=$ $(l / 2, l / 2, m / 2+1)$. It is easy to see that the only partition with this content is $(l, l, m)$. Therefore by Theorem 2.4 and Theorem 4.4 we have $L(A)$ is finitedimensional if and only $\operatorname{part}(\operatorname{RS}(A))=(l, l, m)$ if and only if $A$ is justified rowequivalent to column-strict. Now the statement about the action of $C$ follows from 4.5.

The case where $l$ is odd is similar.

So we are left to consider the case where $l>m$ and $l$ is even. Below we explain the action of $c$ on the s-tables corresponding to finite-dimensional $U(\mathfrak{g}, e)$-modules. We need to use the definition of the $\sharp$-special element of a list of integers, which is given in [Brown 2011, §6].

Let $B \in \mathrm{sTab}^{\leq}\left(P^{\prime}\right)$ be an s-table for some s-frame $P^{\prime}$ with an even number of rows. If the $\sharp$-element of the upper-middle row of $B$ is defined, then we let $c^{\prime} B$ denote the s-table $B^{\prime} \in \mathrm{sTab}^{\leq}\left(P^{\prime}\right)$ where all the rows of $B^{\prime}$ are the same as $B$, except that in the upper-middle row the $\sharp$-element is replaced by its negative, and the corresponding change to the lower-middle row is also made; otherwise we say the $c^{\prime} B$ is undefined.

Let $a_{1}, \ldots, a_{l}$ be the entries in the top row, and let $b_{1}, \ldots, b_{m / 2}$ be the entries in the first half of the middle row of $A$. Let $A^{\prime}$ be the s-table with 4 rows of lengths $l, m / 2, m / 2, l$, where the top row has entries $a_{1}, \ldots, a_{l}$ and the row below the top row has entries $b_{1}, \ldots, b_{m / 2}$.

The rows of $A^{\prime}$ are labelled by $1,2,-2,-1$ from top to bottom. We have the row swapping operators $\bar{s}_{i}$ from Section $4 \mathrm{~B}$ acting on $A^{\prime}$; for convenience in this 
section we do not include the $\star$ in the notation. Let $B=\bar{s}_{1} c^{\prime} \bar{s}_{1} A^{\prime}$, provided that it is defined; otherwise, $c \cdot A$ is undefined.

Let $d_{1}, \ldots, d_{l}$ be the entries in the top row of $B$ and let $e_{1}, \ldots, e_{m}$ be the entries in the row below the top row of $B$. If $e_{1}, \ldots, e_{m}$ are not all negative, then we say that $c \cdot A$ is undefined. Otherwise we declare that $c \cdot A$ is the s-table with row lengths $(l, m, l)$ where the top row has entries $d_{1}, \ldots, d_{l}$ and the middle row has entries $e_{1}, \ldots, e_{m},-e_{m}, \ldots,-e_{1}$

For example, if

$A=$\begin{tabular}{|c|c|c|c|}
\hline 2 & 3 & 4 & 5 \\
\hline-5 & -1 & 1 & \\
\hline-4 & -3 & -2 \\
\hline
\end{tabular}

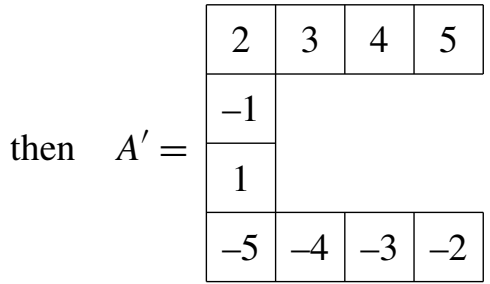

So,

$$
\bar{s}_{1} A^{\prime}=\begin{array}{|r|c|c|c|}
\hline \multicolumn{1}{|c|}{2} & \multicolumn{3}{|c|}{} \\
\hline-1 & 3 & 4 & 5 \\
\hline-5 & -4 & -3 & 1 \\
\hline-2 & \multicolumn{3}{|l}{} \\
\hline
\end{array}
$$

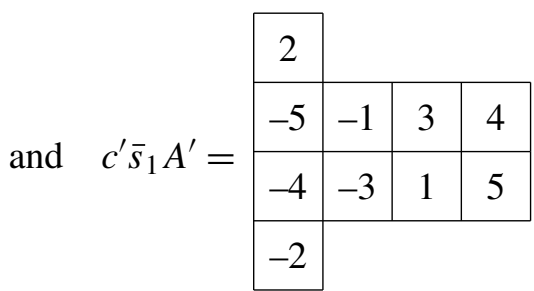

Hence

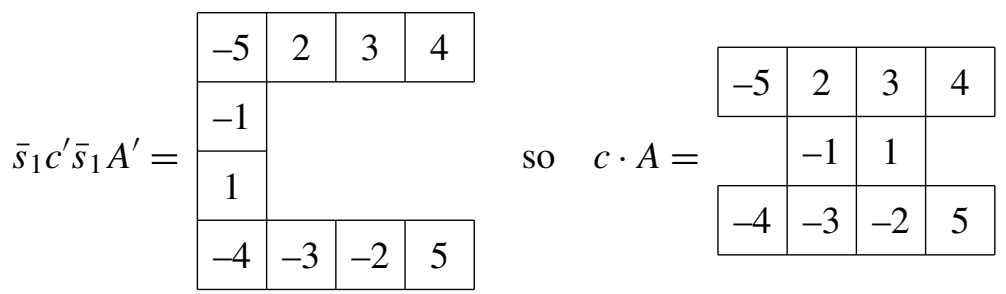

The next lemma follows from [Brown and Goodwin 2013a, Remark 5.8]:

Lemma 5.2. Let $A \in \operatorname{sTab}^{\leq}(P)$ and suppose $c \cdot A$ is defined. Then $\operatorname{word}(A) \sim^{\tau}$ $\operatorname{word}(c \cdot A)$.

Our next goal is to prove that $c \cdot A$ is defined when $A$ corresponds to a finitedimensional $U(\mathfrak{g}, e)$-module:

Lemma 5.3. Let $A \in \mathrm{sTab}^{\leq}(P)$. If $L(A)$ is finite-dimensional, then $c \cdot A$ is defined. Proof. Let $a_{1}, \ldots, a_{l}$ be the top row of $A$ and let $b_{1}, \ldots, b_{m / 2}$ be the first half of the middle row of $A$. Since $L(A)$ is finite-dimensional, we must have that content $(\operatorname{part}(\operatorname{RS}(A)))=\operatorname{content}(l, l, m)=(m / 2, l / 2, l+2 / 2)$. This gives that 
$\operatorname{part}(\operatorname{RS}(A))$ must be $(l, l, m),(l+1, l-1, m)$, or $(l, l-1, m+1)$. The last of these we can rule out by Lemma 4.1. Thus, $\operatorname{part}(\operatorname{RS}(A))=(l, l, m)$ or $(l+1, l-1, m)$. In either case, we note that $\bar{s}_{1} A^{\prime}$ is defined; otherwise we would have for some $i \geq 0$ that $a_{l / 2-i}<b_{m / 2-i}$, in which case we have the increasing subword

$$
a_{1}, \ldots, a_{l / 2-i}, b_{m / 2-i}, \ldots, b_{m / 2},-b_{m / 2}, \ldots,-b_{m / 2-i},-a_{l / 2-i}, \ldots,-a_{1}
$$

of length $l+2$, which contradicts Lemma 4.1.

Now, suppose that $\operatorname{part}(\operatorname{RS}(A))=(l, l, m)$. Then by Theorem 4.4, $A$ is rowequivalent to column-strict, so we have $a_{i}+a_{m-i+1}>0$ for all $i$. Let $a_{1}^{\prime} \leq \cdots \leq a_{m}^{\prime}$ be the elements from the top row that best fit over $b_{1}, \ldots, b_{m / 2},-b_{m / 2}, \ldots,-b_{1}$. Let $a_{1}^{\prime \prime}, \ldots, a_{l-m}^{\prime \prime}$ be the remaining elements of the top row. Then, for $i=1, \ldots, m / 2$ we have that $-a_{m-i+1}^{\prime}<b_{i}<a_{i}^{\prime}$. Since $A$ is row-equivalent to column-strict, we also have that $a_{i}^{\prime \prime}+a_{l-m+1-i}^{\prime \prime}>0$ for all $i$. This shows that the $\sharp$-element of $a_{1}^{\prime \prime}, \ldots, a_{l-m}^{\prime \prime}, b_{1}, \ldots, b_{m / 2}, a_{m / 2+1}^{\prime}, \ldots, a_{m}^{\prime}$ is defined and is greater than or equal to 0 . It also implies that the elements of $\left(a_{1}^{\prime \prime}, \ldots, a_{l-m}^{\prime \prime}, b_{1}, \ldots, b_{m / 2}, a_{m / 2+1}^{\prime}, \ldots, a_{m}^{\prime}\right)^{\#}$ that best fit under $a_{1}^{\prime}, \ldots, a_{m / 2}^{\prime}$ are all negative. Thus $c \cdot A$ is defined.

Now, suppose that part $(\operatorname{RS}(A))=(l+1, l-1, m)$. By [Brown and Goodwin 2013a, Lemma 5.6] the $\sharp$-element of row 2 of $\bar{s}_{1} A^{\prime}$ is defined; otherwise, we could find an increasing subword of length $l+2$. Also, the $\sharp$-element must be negative; otherwise, we could not find an increasing subword of length $l+1 \operatorname{in} \operatorname{word}\left(\bar{s}_{1} A^{\prime}\right)$, since the middle two rows of $\bar{s}_{1} A^{\prime}$ would then be column-strict.

Next, we need to prove that the action of $\bar{s}_{1}$ is defined on $c^{\prime} \bar{s}_{1} A^{\prime}$. If it was not, then we could find two disjoint increasing strings of length $l+1$ in word $\left(c^{\prime} \bar{s}_{1} A^{\prime}\right)$, which is a contradiction since word $\left(c^{\prime} \bar{s}_{1} A^{\prime}\right)$ is $\tau$-equivalent to word $(A)$; compare Theorem 4.9.

Finally, we need to argue why the elements of row 2 of $c^{\prime} \bar{s}_{1} A^{\prime}$ that best fit under row 1 are all negative. If one the best-fitting elements, say $b$, was positive, then we could form the decreasing chain $a, b,-b,-a$, where $a$ is any element of row 1 of $A^{\prime}$ that is larger than $b$. This contradicts the fact that $\operatorname{part}\left(\operatorname{RS}\left(\bar{s}_{1} c^{\prime} \bar{s}_{1} A^{\prime}\right)\right)=(l, l, m)$ or $(l+1, l-1, m)$.

We are now ready for the main theorem of this section:

Theorem 5.4. Suppose that $l$ is even and $l>m$, and let $A \in \mathrm{sTab}^{\leq}(P)$. Then $L(A)$ is finite-dimensional if and only if $A$ is $C$-conjugate to an s-table that is justified row-equivalent to column-strict. Furthermore, if $L(A)$ is finite-dimensional, then $c \cdot L(A) \cong L(c \cdot A)$.

Proof. From the proof of the previous lemma we know that if $L(A)$ is finitedimensional, then $\operatorname{part}(\operatorname{RS}(B))$ is $(l, l, m)$ or $(l+1, l-1, m)$. In the former case, $A$ is row-equivalent to column-strict by Theorem 4.4. In the latter case we can see that $c \cdot A$ is row-equivalent to column-strict immediately from Lemma 5.3 and the 
following observation: Suppose $B \in \operatorname{sTab}^{\leq}(P)$ is such that $\operatorname{part}(\operatorname{RS}(B))=(l, l, m)$ or $(l+1, l-1, m)$ and the middle two rows of $B^{\prime}$ are row-equivalent to column-strict. Then $\operatorname{part}(\operatorname{RS}(B))=(l, l, m)$. Indeed, if we left-justify the top two rows of $B^{\prime}$ and right-justify the bottom two rows then the resulting diagram is column-strict, so it is impossible to find an increasing chain of length $l+1$.

Now we prove the statement about the action of $c$. Suppose that $L(A)$ is finitedimensional and assume that $\operatorname{part}(\operatorname{RS}(A))=(l, l, m)$. We have that $c \cdot L(A) \cong L(B)$ for some $B$. If $\operatorname{part}(\operatorname{RS}(B))=(l, l, m)$, then $A=B$ by Theorem 4.4, and in this case it follows that we have $c \cdot A=B$. If $\operatorname{part}(\operatorname{RS}(B))=(l+1, l-1, m)$, then $\operatorname{part}(\operatorname{RS}(c \cdot B))=(l, l, m)$. So by Lemma 5.2, $L(c \cdot B)$ and $L(A)$ are associated to the same primitive ideal of $U(\mathfrak{g})$. Now from this and the fact that $c \cdot B$ and $A$ are both row-equivalent to column-strict, we can deduce, using Section $2 \mathrm{~B}$ and Theorem 4.5, that $A=c \cdot B$.

Last in this section we give the following lemma, which we need in the proof of Theorem 1.2:

Lemma 5.5. If $A \in \mathrm{sTab}^{\leq}(P)$ is row-equivalent to column-strict, then word $(c \cdot A)$ can be obtained from word $(A)$ through a series of Knuth equivalences and largersmaller transpositions. In particular, $\operatorname{part}(\operatorname{RS}(A)) \leq \operatorname{part}(\operatorname{RS}(c \cdot A))$.

Proof. This is proven in [Brown and Goodwin 2013a, Remark 5.8].

\section{The three row case for $\mathfrak{g}=\mathfrak{s o}_{2 n+1}$}

Let $\mathfrak{g}=\mathfrak{s o}_{2 n}$ and suppose that $\boldsymbol{p}$ has three parts. Then we write $\boldsymbol{p}=\left(l^{2}, m\right)$, where $l$ must be odd if $l>m$. In this section, we classify the finite-dimensional $U(\mathfrak{g}, e)$ modules, and we use the $\tau$-equivalence to describe the component group action on these modules.

Let $C$ be the component group of $e$, so

$$
C= \begin{cases}\langle c\rangle \cong \mathbb{Z}_{2} & \text { if } l \text { is odd and } l \neq m \\ 1 & \text { otherwise }\end{cases}
$$

The lemma below deals with the (easy) cases where $l>m$ (in which case $l$ must be odd) or $l \leq m$ and is even. The proof is very similar to that of Lemma 5.1, so it is omitted.

Lemma 6.1. Suppose that $l$ is even, or $l$ is odd and $l \geq m$. Let $A \in \mathrm{sTab}^{\leq}(P)$. Then $L(A)$ is finite-dimensional if and only if $A$ is justified row-equivalent to columnstrict. Furthermore, in the case that $l$ is odd and $l>m$, if $L(A)$ is finite-dimensional, then $c \cdot L(A) \cong L(A)$.

So we are left to consider the case where $l$ is odd and $m>l$; in this case, we let $l=2 p+1$ and $m=2 q+1$, where $q>p$. In the next few paragraphs we set up the combinatorics to describe the action of $c$ on elements of $\mathrm{sTab}^{\leq}(P)$ corresponding 
to finite-dimensional representations.

Let $A \in \mathrm{sTab}^{\leq}(P)$. Let $a_{1}, \ldots, a_{2 p+1}$ be the top row of $A$ and let $b_{1}, \ldots, b_{q}, 0$, $-b_{q}, \ldots,-b_{1}$ be the middle row. From $A$, we define two tables, $A^{L^{+}}$and $A^{L^{-}}$, in the following manner: $A^{L^{+}}$is the left-justified three row table with row 1 equal to $a_{1}, \ldots, a_{p+1}$, row 2 equal to $b_{1}, \ldots, b_{q}$, and row 3 equal to $-a_{2 p+1}, \ldots,-a_{p+2}$. $A^{L^{-}}$is the left-justified three row table with row 1 equal to $a_{1}, \ldots, a_{p}$, row 2 equal to $b_{1}, \ldots, b_{q}$, and row 3 equal to $-a_{2 p+1}, \ldots,-a_{p+1}$.

We define the $C$-action on $A$ in the following manner depending on the cases below. Here we use the row swapping operations for tables mentioned in Section 4B, and we omit the $\star$ in the notation for convenience.

Case 1: If $A^{L^{-}}$is row-equivalent to column-strict, then we define $c \cdot A=B$, where $B$ is the unique s-table in $\mathrm{sTab}^{\leq}(P)$ such that $B^{L^{+}}=s_{2} s_{1} s_{2} A^{L^{-}}$.

Case 2: If $A^{L^{-}}$is not row-equivalent to column-strict but $A^{L^{+}}$is row-equivalent to column-strict, then we define $c \cdot A=B$, where $B$ is the unique s-table in sTab ${ }^{\leq}(P)$ such that $B^{L^{-}}=s_{2} s_{1} s_{2} A^{L^{+}}$, provided that such an s-table exists; note that $B$ exists precisely when $s_{1} s_{2} A^{L^{+}}$contains only negative numbers in row 2 , and this will not happen if $A^{L^{-}}$is row-equivalent to column-strict. If such a $B$ does not exist, then we say that $c \cdot A$ is not defined.

Case 3: If neither $A^{L^{-}}$nor $A^{L^{+}}$is row-equivalent to column-strict, then we say that $c \cdot A$ is undefined.

For example, suppose that

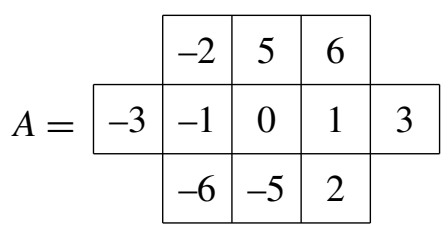

Then

$$
A^{L^{+}}=\begin{array}{r|r|}
\hline-2 & 5 \\
\hline-3 & -1 \\
\hline-6 &
\end{array} \quad \text { and } \quad A^{L^{-}}=\begin{array}{r|r|r|}
-2 & \\
\hline-3 & -1 \\
\hline-6 & -5 \\
\hline
\end{array}
$$

Since $A^{L^{-}}$is column-strict, we are in Case 1 . Now

$$
s_{2} s_{1} s_{2} A^{L^{-}}=\begin{array}{|r|r|}
\hline-2 & -1 \\
\hline-6 & -3 \\
\hline-5 &
\end{array}
$$


so

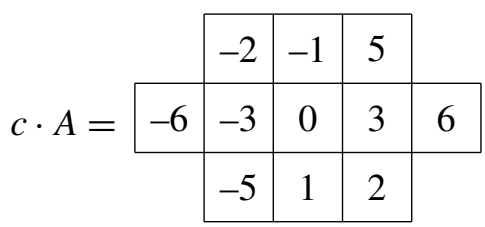

We need to prove that word $(A)$ is $\tau$-equivalent to $\operatorname{word}(c \cdot A)$. To do this, we need the following lemmas:

Lemma 6.2. Let $a, b_{1}, \ldots, b_{m}$ be such that $a>0, b_{1}<\cdots<b_{m}<0$ and $-a<b_{m}$. Then

$$
\left(a, b_{1}, \ldots, b_{m}\right) \sim^{\tau}\left(b_{1}, \ldots, b_{m},-a\right) .
$$

Proof. By applying the Robinson-Schensted algorithm we see that $\left(a, b_{1}, \ldots, b_{m}\right)$ is Knuth-equivalent to $\left(b_{1}, \ldots, b_{m-1}, a, b_{m}\right)$. By applying the relations (R3) then (R2) from the definition of the $\tau$-equivalence, we get that this is $\tau$-equivalent to $\left(b_{1}, \ldots, b_{m},-a\right)$.

For positive integers $k, m$, we define an operation $\mathrm{LT}_{k, m}$ on certain lists. Suppose that $\left(a_{1}, \ldots, a_{l}, b_{1}, \ldots, b_{m}\right)$ is a list such that $l \geq 2 k-1, m \geq k, b_{m}<0, a_{l-k}>0$, and the table

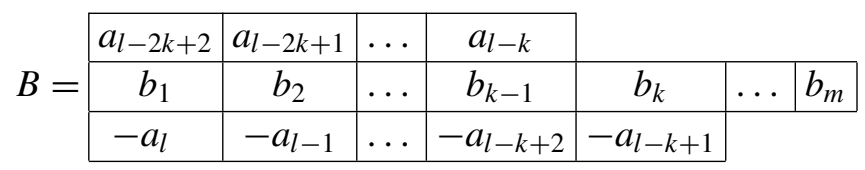

is row-equivalent to column-strict with increasing rows. We define

$$
\mathrm{LT}_{k, m}\left(a_{1}, \ldots, a_{l}, b_{1}, \ldots, b_{m}\right)
$$

to be the list $\left(a_{1}, \ldots, a_{l-2 k+1}\right)$ concatenated with word $(B)$. For example, if $A \in$ $\mathrm{sTab}^{\leq}(P)$ is justified row-equivalent to column-strict, $P$ has row lengths $(2 p+$ $1,2 q+1,2 p+1)$, and

$\operatorname{word}(A)=\left(a_{1}, \ldots, a_{2 p+1}, b_{1}, \ldots, b_{2 q+1}, 0,-b_{2 q+1}, \ldots,-b_{1},-a_{2 p+1}, \ldots,-a_{1}\right)$, then $\mathrm{LT}_{p+1,2 q+1}\left(a_{1}, \ldots, a_{2 p+1}, b_{1}, \ldots, b_{2 q+1}\right)=\operatorname{word}\left(A^{L^{-}}\right)$.

We would also like to explicitly describe $\mathrm{LT}_{k, m}^{-1}$. This will be defined on lists of the form $\left(a_{1}, \ldots, a_{l}, b_{1}, \ldots, b_{m}, c_{1}, \ldots, c_{k}\right)$, where $m \geq k, l \geq k-1, c_{k}<0$, $-c_{k}>a_{l}, b_{m}<0$, and the following table is row-equivalent to column-strict with increasing rows:

$$
B=\begin{array}{|c|c|c|c|c|c|c|}
\hline a_{l-k+2} & a_{l-k+1} & \ldots & a_{l} & \multicolumn{3}{|l}{} \\
\hline b_{1} & b_{2} & \ldots & b_{k-1} & b_{k} & \ldots & b_{m} \\
\hline c_{1} & c_{2} & \ldots & c_{k-1} & c_{k} & &
\end{array}
$$


Now

$\mathrm{LT}_{k, m}^{-1}\left(a_{1}, \ldots, a_{l}, c_{1}, \ldots, c_{k}, b_{1}, \ldots, b_{m}\right)=\left(a_{1}, \ldots, a_{l},-c_{k}, \ldots,-c_{1}, b_{1}, \ldots, b_{m}\right)$.

We say that $\mathrm{LT}_{k, m}^{-1}\left(a_{1}, \ldots, a_{l}, b_{1}, \ldots, b_{m}, c_{1}, \ldots, c_{k}\right)$ is undefined if any of the above conditions is not met.

Lemma 6.5. Let $\left(a_{1}, \ldots, a_{l}, b_{1}, \ldots, b_{m}\right)$ be a list on which $\mathrm{LT}_{k, m}$ is defined. Then

$$
\left(a_{1}, \ldots, a_{l}, b_{1}, \ldots, b_{m}\right) \sim^{\tau} \operatorname{LT}_{k, m}\left(a_{1}, \ldots, a_{l}, b_{1}, \ldots, b_{m}\right) .
$$

Proof. We may assume that $l=2 k-1$. We proceed by induction on $k$. The case $k=1$ is given by Lemma 6.2. Now, since

\begin{tabular}{|c|c|c|c|c|c|c|}
\hline$a_{1}$ & $a_{2}$ & $\ldots$ & $a_{k-1}$ & \multicolumn{3}{|c|}{} \\
\hline$b_{1}$ & $b_{2}$ & $\ldots$ & $b_{k-1}$ & $b_{k}$ & $\ldots$ & $b_{m}$ \\
\hline$-a_{2 k-1}$ & $-a_{2 k-2}$ & $\ldots$ & $-a_{k+1}$ & $-a_{k}$ & \multicolumn{1}{|c}{} \\
\cline { 1 - 4 } & & &
\end{tabular}

is row-equivalent to column-strict, we also have that

\begin{tabular}{|c|c|c|c|c|c|c|}
\hline$a_{3}$ & $a_{4}$ & $\ldots$ & $a_{k}$ & \multicolumn{3}{|c|}{} \\
\hline$b_{1}$ & $b_{2}$ & $\ldots$ & $b_{k-2}$ & $b_{k-1}$ & $\ldots$ & $b_{m}$ \\
\hline$-a_{2 k-1}$ & $-a_{2 k-2}$ & $\ldots$ & $-a_{k+2}$ & $-a_{k+1}$ & \multicolumn{2}{|c}{} \\
\cline { 1 - 4 } & &
\end{tabular}

is row-equivalent to column-strict. So by induction $\left(a_{1}, \ldots, a_{l}, b_{1}, \ldots, b_{m}\right)$ is $\tau$ equivalent to $\operatorname{LT}_{k-1, m}\left(a_{1}, \ldots, a_{l}\right)=\left(a_{1}, \ldots, a_{k}, b_{1}, \ldots, b_{m},-a_{2 k-1}, \ldots,-a_{k+1}\right)$. Now let $b_{i_{1}}, \ldots, b_{i_{k-1}}$ be the elements of $b_{1}, \ldots, b_{m}$ that best fit over $-a_{2 k-1}, \ldots$, $-a_{k+1}$. Thus

$$
\begin{aligned}
\left(a_{1}, \ldots, a_{k}, b_{1}, \ldots, b_{m},-a_{2 k-1}\right. & \left., \ldots,-a_{k+1}\right) \\
& { }^{K}\left(a_{1}, \ldots, a_{k}, b_{i_{1}}, \ldots, b_{i_{k-1}}, a_{1}^{\prime}, \ldots, a_{m}^{\prime}\right),
\end{aligned}
$$

where $\sim^{K}$ denotes Knuth equivalence and $\left(a_{1}^{\prime}, \ldots, a_{m}^{\prime}\right)$ is the sorted list consisting of $-a_{2 k-1}, \ldots,-a_{k+1}$ and $\left\{b_{l} \mid l \neq i_{j}\right.$ for $\left.j=1, \ldots, k-1\right\}$. Now, from (6.6) we can see that $b_{i_{1}}, \ldots, b_{i_{k-1}}$ best fits under $a_{1}, \ldots, a_{k-1}$, so

$$
\begin{aligned}
\left(a_{1}, \ldots, a_{k}, b_{i_{1}}, \ldots, b_{i_{k-1}}, a_{1}^{\prime}, \ldots, a_{m}^{\prime}\right) & \\
& \sim^{K}\left(a_{1}, \ldots, a_{k-1}, b_{i_{1}}, \ldots, b_{i_{k-1}}, a_{k}, a_{1}^{\prime}, \ldots, a_{m}^{\prime}\right) .
\end{aligned}
$$

We also get from (6.6) that $a_{m}^{\prime}=-b_{m}$, so by Lemma 6.2 we have that

$$
\begin{aligned}
\left(a_{1}, \ldots, a_{k-1}, b_{i_{1}}, \ldots, b_{i_{k-1}}, a_{k},\right. & \left.a_{1}^{\prime}, \ldots, a_{m}^{\prime}\right) \\
& \sim^{\tau}\left(a_{1}, \ldots, a_{k-1}, b_{i_{1}}, \ldots, b_{i_{k-1}}, a_{1}^{\prime}, \ldots, a_{m}^{\prime},-a_{k}\right) .
\end{aligned}
$$

Finally we can use the Knuth equivalence in (6.7) to get that this is Knuth-equivalent to

$$
\left(a_{1}, \ldots, a_{k-1}, b_{1}, \ldots, b_{m},-a_{2 k-1}, \ldots,-a_{k}\right)
$$


Lemma 6.8. Suppose that we are given a skew-symmetric word

$$
w=\left(a, b_{1}, \ldots, b_{m}, c, 0,-c,-b_{m}, \ldots,-b_{1},-a\right)
$$

such that $\operatorname{part}\left(\operatorname{RS}\left(a, b_{1}, \ldots, b_{m}, c\right)\right)=(m, 1,1), b_{1}<b_{2}<\cdots<b_{m}<0, c<0$, and $-c>a$. Then

$$
w \sim^{K}\left(a, b_{1}, \ldots, b_{m},-c, 0, c,-b_{m}, \ldots,-b_{1},-a\right) .
$$

Proof. Calculate

$\mathrm{RS}\left(a, b_{1}, \ldots, b_{m}, c, 0,-c,-b_{m}\right) \quad$ and $\quad \mathrm{RS}\left(a, b_{1}, \ldots, b_{m},-c, 0, c,-b_{m}\right)$, then observe that they are equal.

Lemma 6.9. Suppose that we are given a skew-symmetric word $w=\left(a_{1}, \ldots, a_{l}, b_{1}, \ldots, b_{m}, c_{1}, \ldots, c_{k}, 0,-c_{k}, \ldots,-c_{1},-b_{m}, \ldots,-b_{1},-a_{l}, \ldots,-a_{1}\right)$ such that $k \leq l \leq m, a_{1}<a_{2}<\cdots<a_{l}, b_{1}<b_{2}<\cdots<b_{m}<0, c_{1}<c_{2}<\cdots<c_{k}<0$, $-c_{k}>a_{l}$, and $\operatorname{part}\left(\operatorname{RS}\left(a_{1}, \ldots, a_{l}, b_{1}, \ldots, b_{m}, c_{1}, \ldots, c_{k}\right)\right)=(m, l, k)$. Then $w \sim^{K}\left(a_{1}, \ldots, a_{l}, b_{1}, \ldots, b_{m},-c_{k}, \ldots,-c_{1}, 0, c_{1}, \ldots, c_{k},-b_{m}, \ldots,-b_{1},-a_{l}, \ldots,-a_{1}\right)$ and

$$
\left(a_{1}, \ldots, a_{l}, b_{1}, \ldots, b_{m}, c_{1}, \ldots, c_{k}\right) \sim^{\tau}\left(a_{1}, \ldots, a_{l}, b_{1}, \ldots, b_{m},-c_{k}, \ldots,-c_{1}\right) .
$$

Proof. We prove this by induction on $k$. The case $k=1$ is given by Lemma 6.8 and condition (R2) in the definition of the $\tau$-equivalence.

To prove the general case, first we best-fit $c_{1}, \ldots, c_{k-1}$ under $b_{1}, \ldots, b_{m}$, which gives that

$$
\left(b_{1}, \ldots, b_{m}, c_{1}, \ldots, c_{k-1}\right) \sim^{K}\left(b_{i_{1}}, \ldots, b_{i_{k-1}}, c_{1}^{\prime}, \ldots, c_{m}^{\prime}\right) .
$$

Now we can best fit $b_{i_{1}}, \ldots, b_{i_{k-1}}$ under $a_{1}, \ldots, a_{l}$ to get that

$$
\left(a_{1}, \ldots, a_{l}, b_{i_{1}}, \ldots, b_{i_{k-1}}\right) \sim^{K}\left(a_{i_{1}^{\prime}}, \ldots, a_{i_{k-1}^{\prime}}, b_{1}^{\prime}, \ldots, b_{l}^{\prime}\right) .
$$

Putting this all together, we get that $w$ is Knuth-equivalent to

$$
\begin{aligned}
& \left(a_{i_{1}^{\prime}}, \ldots, a_{i_{k-1}^{\prime}}, b_{1}^{\prime}, \ldots, b_{l}^{\prime}, c_{1}^{\prime}, \ldots, c_{m}^{\prime}, c_{k}, 0,\right. \\
& \left.\quad-c_{k},-c_{m}^{\prime}, \ldots,-c_{1}^{\prime},-b_{l}^{\prime}, \ldots,-b_{1}^{\prime},-a_{i_{k-1}^{\prime}}^{\prime}, \ldots,-a_{i_{1}^{\prime}}\right) .
\end{aligned}
$$

Since $\operatorname{part}\left(\operatorname{RS}\left(a_{1}, \ldots, a_{l}, b_{1}, \ldots, b_{m}, c_{1}, \ldots, c_{k}\right)\right)=(m, l, k)$, we can deduce that $b_{l}^{\prime}=a_{l}$ and $c_{m}^{\prime}=b_{m}$. We can also use this to deduce that the element of $\left(b_{1}, \ldots, b_{m}\right)$ that best fits over $c_{k}$ is an element of $\left(c_{1}^{\prime}, \ldots, c_{m}^{\prime}\right)$. Now, we apply 
Lemma 6.8 to the part of this word between $b_{l}^{\prime}$ and $-b_{l}^{\prime}$ to get that this is Knuthequivalent to

$$
\begin{array}{r}
\left(a_{i_{1}^{\prime}}, \ldots, a_{i_{k-1}^{\prime}}, b_{1}^{\prime}, \ldots, b_{l}^{\prime}, c_{1}^{\prime}, \ldots, c_{m}^{\prime},-c_{k}, 0,\right. \\
\left.c_{k},-c_{m}^{\prime}, \ldots,-c_{1}^{\prime},-b_{l}^{\prime}, \ldots,-b_{1}^{\prime},-a_{i_{k-1}^{\prime}}^{\prime}, \ldots,-a_{i_{1}^{\prime}}\right) .
\end{array}
$$

We can apply the Knuth equivalences in (6.10) and (6.11) to get that this is Knuthequivalent to

$$
\begin{aligned}
\left(a_{1}, \ldots, a_{l}, b_{1}, \ldots, b_{m}, c_{1}, \ldots, c_{k-1},-c_{k}, 0,\right. \\
\\
\left.c_{k},-c_{k-1}, \ldots,-c_{1},-b_{m}, \ldots,-b_{1},-a_{l}, \ldots,-a_{1}\right) .
\end{aligned}
$$

Now, we can best fit $b_{m-k+2}, \ldots, b_{m}$ over $c_{1}, \ldots, c_{k-1},-c_{k}$ to get

$$
\left(b_{m-k+2}, \ldots, b_{m}, c_{1}, \ldots, c_{k-1},-c_{k}\right) \sim^{K}\left(b_{m-k+2}, \ldots, b_{m},-c_{k}, c_{1}, \ldots, c_{k-1}\right) .
$$

Next, we can best fit $a_{1}, \ldots, a_{l}$ over $b_{1}, \ldots, b_{m},-c_{k}$ to get that

$$
\left(a_{1}, \ldots, a_{l}, b_{1}, \ldots, b_{m},-c_{k}\right) \sim^{K}\left(a_{1}^{\prime}, \ldots, a_{m}^{\prime},-c_{k}, b_{j_{1}}, \ldots, b_{j_{l}}\right) .
$$

So we have that

$$
\begin{array}{r}
\left(a_{1}, \ldots, a_{l}, b_{1}, \ldots, b_{m}, c_{1}, \ldots, c_{k-1},-c_{k}, 0,\right. \\
\left.c_{k},-c_{k-1}, \ldots,-c_{1},-b_{m}, \ldots,-b_{1},-a_{l}, \ldots,-a_{1}\right),
\end{array}
$$

and therefore $w$, is Knuth-equivalent to

$$
\begin{aligned}
\left(a_{1}^{\prime}, \ldots, a_{m}^{\prime},-c_{k}, b_{j_{1}}, \ldots, b_{j_{l}}, c_{1}, \ldots, c_{k-1}, 0,\right. & \\
& \left.\quad-c_{k-1}, \ldots,-c_{1},-b_{j_{l}}, \ldots,-b_{j_{1}}, c_{k},-a_{m}^{\prime}, \ldots,-a_{1}^{\prime}\right) .
\end{aligned}
$$

By induction this is Knuth-equivalent to

$$
\begin{aligned}
\left(a_{1}^{\prime}, \ldots, a_{m}^{\prime},-c_{k}, b_{j_{1}}, \ldots, b_{j_{l}},-c_{k-1}, \ldots,-c_{1}, 0,\right. \\
\left.c_{1}, \ldots, c_{k-1},-b_{j_{l}}, \ldots,-b_{j_{1}}, c_{k},-a_{m}^{\prime}, \ldots,-a_{1}^{\prime}\right) .
\end{aligned}
$$

Finally, by applying the Knuth equivalence (6.12), we get that this is Knuthequivalent to

$\left(a_{1}, \ldots, a_{l}, b_{1}, \ldots, b_{m},-c_{k}, \ldots,-c_{1}, 0, c_{1}, \ldots, c_{k},-b_{m}, \ldots,-b_{1},-a_{l}, \ldots,-a_{1}\right)$.

Theorem 6.13. Let $A \in \mathrm{sTab}^{\leq}(P)$ be justified row-equivalent to column-strict. Let

$$
\left(a_{1}, \ldots, a_{q+1}, b_{1}, \ldots, b_{p},-a_{2 q+1}, \ldots,-a_{q+2}\right)=\operatorname{word}\left(s_{2} s_{1} s_{2} A^{L^{-}}\right) .
$$

Then

$$
\begin{aligned}
& \left(a_{1}, \ldots, a_{q+1}, b_{1}, \ldots, b_{p},-a_{2 q+1}, \ldots,-a_{q+2}, 0,\right. \\
& \left.a_{q+2}, \ldots, a_{2 q+1},-b_{p}, \ldots,-b_{1},-a_{q+1}, \ldots,-a_{1}\right)
\end{aligned}
$$


is Knuth-equivalent to $\operatorname{word}(c \cdot A)$. In particular, this implies that $\operatorname{word}(A)$ is $\tau$-equivalent to $\operatorname{word}(c \cdot A)$.

Proof. By Lemma 6.9 we have that

$$
\begin{aligned}
& \left(a_{1}, \ldots, a_{q+1}, b_{1}, \ldots, b_{p},-a_{2 q+1}, \ldots,-a_{q+2}, 0,\right. \\
& \left.\quad a_{q+2}, \ldots, a_{2 q+1},-b_{p}, \ldots,-b_{1},-a_{q+1}, \ldots,-a_{1}\right)
\end{aligned}
$$

is Knuth-equivalent to

$$
\begin{aligned}
\left(a_{1}, \ldots, a_{q+1}, b_{1}, \ldots, b_{p},\right. & a_{q+2}, \ldots, a_{2 q+1}, 0, \\
& \left.-a_{2 q+1}, \ldots,-a_{q+2},-b_{p}, \ldots,-b_{1},-a_{q+1}, \ldots,-a_{1}\right) .
\end{aligned}
$$

Now, if $b_{i_{1}}, \ldots, b_{i_{q+1}}$ best fits under $a_{1}, \ldots, a_{q+1}$, then we get that this is Knuthequivalent to

$$
\begin{aligned}
& \left(a_{1}^{\prime}, \ldots, a_{p}^{\prime}, a_{q+2}, \ldots, a_{2 q+1}, b_{i_{1}}, \ldots, b_{i_{q+1}}, 0,\right. \\
& \left.\quad-b_{i_{q+1}}, \ldots,-b_{i_{1}},-a_{2 q+1}, \ldots,-a_{q+2},-a_{p}^{\prime}, \ldots,-a_{1}^{\prime}\right) .
\end{aligned}
$$

Note that $a_{p}^{\prime}=a_{q+1}$ or $a_{p}^{\prime}=b_{j}<0$ for some $j$, so in either case we can best fit

$$
b_{i_{1}}, \ldots, b_{i_{q+1}}, 0,-b_{i_{q+1}}, \ldots,-b_{i_{3}} \quad \text { under } \quad a_{1}^{\prime}, \ldots, a_{p}^{\prime}, a_{q+2}, \ldots, a_{2 q+1}
$$

to get that

$$
\begin{aligned}
& \left(a_{1}^{\prime}, \ldots, a_{p}^{\prime}, a_{q+2}, \ldots, a_{2 q+1}, b_{i_{1}}, \ldots, b_{i_{q+1}}, 0,\right. \\
& \left.\quad-b_{i_{q+1}}, \ldots,-b_{i_{1}},-a_{2 q+1}, \ldots,-a_{q+2},-a_{p}^{\prime}, \ldots,-a_{1}^{\prime}\right)
\end{aligned}
$$

is Knuth-equivalent to

$$
\left(a_{1}, \ldots, a_{2 q+1}, b_{1}, \ldots, b_{m}, 0,-b_{i_{q+1}}, \ldots,-b_{i_{1}},-a_{2 q+1}, \ldots,-a_{q+2},-a_{p}^{\prime}, \ldots,-a_{1}^{\prime}\right) \text {. }
$$

Now we can best fit

$b_{m-q+2}, \ldots, b_{m}, 0,-b_{i_{q+1}}, \ldots,-b_{i_{1}} \quad$ over $\quad-a_{2 q+1}, \ldots,-a_{q+2},-a_{p}^{\prime}, \ldots,-a_{1}^{\prime}$ to get that

$$
\left(a_{1}, \ldots, a_{2 q+1}, b_{1}, \ldots, b_{m}, 0,-b_{i_{q+1}}, \ldots,-b_{i_{1}},-a_{2 q+1}, \ldots,-a_{q+2},-a_{p}^{\prime}, \ldots,-a_{1}^{\prime}\right)
$$

is Knuth-equivalent to

$$
\left(a_{1}, \ldots, a_{2 q+1}, b_{1}, \ldots, b_{m}, 0,-b_{m}, \ldots,-b_{1},-a_{2 q+1}, \ldots,-a_{1}\right) .
$$

Our goal is to prove that $L(A)$ is finite-dimensional if and only if $A$ is $C$-conjugate to a row-equivalent to column-strict diagram. The following lemmas build up to this:

Lemma 6.14. Let $A \in \mathrm{sTab}^{\leq}(P)$. If $A^{L^{-}}$is row-equivalent to column-strict, then so is $A$. 
Proof. Recall that $A$ has row lengths given by $(2 p+1,2 q+1,2 p+1)$. By permuting entries within rows, we can find $p$ strictly decreasing columns of $A^{L^{-}}$Furthermore, the entry in the bottom row of $A^{L^{-}}$that is not in one of these columns must be negative. By putting this entry below 0 in $A$ and its negation above 0 , we can find a row equivalence class of $A$ where every column left of 0 contains one of the decreasing columns from $A^{L^{-}}$, and every column right of zero is the reverse of the negation of one of the columns left of 0 . Thus, every column in this element of the row equivalence class of $A$ is strictly decreasing.

Lemma 6.15. Let $A \in \operatorname{sTab}^{\leq}(P)$, and let $\boldsymbol{q}=\operatorname{part}(\operatorname{RS}(A))$. If $\operatorname{content}(\boldsymbol{q})=$ content $(\boldsymbol{p})$, then $\boldsymbol{q}=(2 q+1,2 p+1,2 p+1)$ or $\boldsymbol{q}=(2 q+1,2 p+2,2 p)$.

Proof. Note that content $(2 q+1,2 p+1,2 p+1)=(p, p+1, q+1)$, and the only other partition with this content is $(2 q, 2 p+2,2 p+1)$. Now, by Lemma 4.1, $\operatorname{part}(\operatorname{RS}(A)) \geq(2 q+1,2 p+1,2 p+1), \operatorname{thus} \operatorname{part}(\operatorname{RS}(A)) \neq(2 q, 2 p+2,2 p+1)$.

By Theorem 4.4 we have that if $\operatorname{part}(\operatorname{RS}(A))=(2 q+1,2 p+1,2 p+1)$, then $A$ is row-equivalent to column-strict. So we need only consider the case that $\operatorname{part}(\operatorname{RS}(A))=(2 q+1,2 p+2,2 p)$.

Lemma 6.16. Let $A \in \operatorname{sTab}^{\leq}(P)$ with $\operatorname{part}(\operatorname{RS}(A))=(2 q+1,2 p+2,2 p)$. Then:

(1) $A^{L^{+}}$is row-equivalent to column-strict.

(2) The middle row of $s_{2} s_{1} s_{2} A^{L^{+}}$contains only negative numbers.

(3) The negation of the element in the bottom-right position of $s_{2} s_{1} s_{2} A^{L^{+}}$is larger than the element in the upper-right position of $s_{2} s_{1} s_{2} A^{L^{+}}$. Thus $c \cdot A$ is defined.

(4) $c \cdot A$ is row-equivalent to column-strict.

Proof. Let $a_{-p}, \ldots, a_{-1}, a_{0}, a_{1}, \ldots, a_{p}$ be the increasing entries in the first row of $A$, and let $-b_{q}, \ldots,-b_{1}, 0, b_{1}, \ldots, b_{q}$ be the middle row of $A$.

First we prove that $a_{-p}, \ldots, a_{0}$ must best fit over $-b_{q}, \ldots,-b_{1}$. If it does not, then there must exists $i \in\{0, \ldots, p\}$ such that $a_{-(p-i)}<-b_{q-i}$. Thus we can form the following increasing string in $\operatorname{word}(A)$ :

$$
a_{-p}, \ldots, a_{-(p-i)},-b_{q-i}, \ldots,-b_{1}, 0, b_{1}, \ldots, b_{q-i},-a_{-(p-i)}, \ldots,-a_{-p} .
$$

This string has length $2 q+3$, which contradicts part $(\operatorname{RS}(A))=(2 q+1,2 p+2,2 p)$.

Next we prove that $a_{1}, \ldots, a_{p}$ best fits over $b_{1}, \ldots, b_{q}$. If it does not, then there exists $i \in\{1, \ldots, p\}$ such that $a_{i}<b_{i}$. Thus we can form the following increasing string in $\operatorname{word}(A)$ :

$$
a_{-p}, \ldots, a_{0}, \ldots, a_{i}, b_{i}, \ldots, b_{q} .
$$

This string has length $p+q+2$, and we can use it to find the following increasing string of length $2 p+2 q+4$ in $\operatorname{word}(A)$ :

$$
a_{-p}, \ldots, a_{0}, \ldots, a_{i}, b_{i}, \ldots, b_{q},-b_{q}, \ldots,-b_{i},-a_{1}, \ldots,-a_{0}, \ldots,-a_{-p} .
$$


This contradicts part $(\operatorname{RS}(A))=(2 q+1,2 p+2,2 p)$.

Now, we assume for a contradiction that $A^{L^{+}}$is not row-equivalent to columnstrict. Let $j_{0}, \ldots, j_{p}$ be positive integers such that $-b_{j_{p}}, \ldots,-b_{j_{0}}$ best fit under $a_{-p}, \ldots, a_{0}$. Let $i$ be the smallest nonnegative integer such that $-b_{j_{i}}<-a_{i+1}$. Such an $i$ must exist, since otherwise $A^{L^{+}}$will be row-equivalent to column-strict. Define $b_{0}=0$. Now let $k$ be the smallest integer such that

(1) $0 \leq k \leq i$;

(2) $j_{i-l}=j_{i}-l$ if $0<l \leq k$;

(3) $j_{i-k-1} \neq j_{i-k}-1$.

This implies that $a_{-(i-k)}<-b_{j_{i-k}-1}$. So we can form the following two disjoint increasing substrings in $\operatorname{word}(A)$ :

$$
a_{-p}, \ldots, a_{-(i-k)},-b_{j_{i-k}-1}, \ldots, b_{-1}, 0, b_{1}, \ldots, b_{q}
$$

and

$$
-b_{q}, \ldots,-b_{j_{i}},-a_{i+1}, \ldots,-a_{1},-a_{0},-a_{-1}, \ldots,-a_{-p} .
$$

The first string has length $p-i+k+1+j_{i-k}-1+1+q=p+q-i+k+j_{i-k}+1$. The second string has length $q-j_{i}+1+i+1+p+1=q+p-j_{i}+i+3$. Thus, using the fact that $j_{i-k}=j_{i}-k$, the combined length of these two strings is $2 q+2 p+4$, which contradicts $\operatorname{part}(\operatorname{RS}(A))=(2 q+1,2 p+2,2 p)$. Thus $A^{L^{+}}$is row-equivalent to column-strict.

Finally we need to prove that the middle row of $s_{2} s_{1} s_{2} A^{L^{+}}$contains only negative numbers. Let $j_{1}, \ldots, j_{p}$ be such that $-b_{j_{p}}, \ldots,-b_{j_{1}}$ best fit over $-a_{p}, \ldots,-a_{1}$. Now it is clear that all the numbers in the last row of $s_{2} A^{L^{+}}$are negative. Now let $a^{\prime}$ be the entry in the first row of $A^{L^{+}}$that does not best fit over $-b_{j_{p}}, \ldots,-b_{j_{1}}$. If $a^{\prime}>0$, then since all the $-b_{i}$ are negative we must have that $a^{\prime}=a_{0}$. In this case, for $i=1, \ldots, p,\left(a_{-i},-b_{j_{i}},-a_{i}\right)$ is a decreasing string in word $\left(A^{L^{+}}\right)$and in $\operatorname{word}(A)$. Furthermore, reversing and negating these strings yields a further $p$ disjoint deceasing strings of length 3 in word $(A)$. These and the string $\left(a_{0}, 0,-a_{0}\right)$ show that $\operatorname{part}(\operatorname{RS}(A))^{T}$ is larger than a partition of the form $\left(3^{2 p+1}, *\right)$. This contradicts $\operatorname{part}(\operatorname{RS}(A))=(2 q+1,2 p+2,2 p)$. So we have that $a^{\prime}<0$, and furthermore the middle row of $s_{1} s_{2} A^{L^{+}}$contains only negative numbers. Now since the last row of $s_{1} s_{2} A^{L^{+}}$also contains all negative numbers, we have that the middle row of $s_{2} s_{1} s_{2} A^{L^{+}}$contains only negative numbers.

Now, let $x$ be the element in the upper-right position of $s_{2} s_{1} s_{2} A^{L^{+}}$and let $y$ be the element in the lower-right position. We need to show that $x<-y$. If $a_{0}<0$ then this is clear, since in this case every element of $A^{L^{+}}$is negative. When $a_{0}>0$, we need to consider the bottom row of $s_{2} s_{1} s_{2} A^{L^{+}}$. This row will contain $-a_{n}, \ldots,-a_{1}$ and also $-b_{i}$, where $-b_{i}$ is not one of the elements of $-b_{m}, \ldots,-b_{1}$ that best fits 
over $-a_{n}, \ldots,-a_{1}$. Let $-b_{k_{n}}, \ldots,-b_{k_{1}}$ be as above, i.e., the elements which best fit over $-a_{n}, \ldots,-a_{1}$. Note that $-b_{k_{n}}, \ldots,-b_{k_{1}}$ are the elements in the middle row of $s_{2} A^{L^{+}}$. Now, let $a^{\prime}$ be the element in the first row of $A^{L^{+}}$that is one of the elements which best fit over $-b_{k_{n}}, \ldots,-b_{k_{1}}$, so the middle row of $s_{1} s_{s} A^{L^{+}}$ contains $-a^{\prime},-b_{k_{n}}, \ldots,-b_{k_{1}}$. We have already proved that since $a_{0}>0, a^{\prime}<0$. So $a^{\prime}<-b_{k_{1}}$, since otherwise $a_{0}$ would be the element that did not best fit over $-b_{k_{n}}, \ldots,-b_{k_{1}}$. So $-b_{i}<a^{\prime}<-b_{k_{1}}$. This implies that $-b_{i}<-a_{1}$, since otherwise $-b_{k_{1}}$ would not be the element that best fits over $-a_{1}$. Thus $-a_{1}$ is the element in the bottom-right position of $s_{2} s_{1} s_{2} A^{L^{+}}$, and $a_{0}$ is the element in the upper-right position of $s_{2} s_{1} s_{2} A^{L^{+}}$, and we already have that $a_{0}<a_{1}$.

To see that $c \cdot A$ is row-equivalent to column-strict, simply note that $(c \cdot A)^{L^{-}}=$ $s_{2} s_{1} s_{2} A^{L^{+}}$is row-equivalent to column-strict and apply Lemma 6.14.

Now we can state the main theorem of this section, which is analogous to Theorem 5.4. The proof is very similar, where Lemma 6.16 plays the role of Lemma 5.3, and so is omitted.

Theorem 6.17. Suppose that $l$ is odd and $l>m$, and let $A \in \mathrm{sTab}^{\leq}(P)$. Then $L(A)$ is finite-dimensional if and only if $A$ is $C$-conjugate to an s-table that is justified row-equivalent to column-strict. Furthermore, if $L(A)$ is finite-dimensional, then $c \cdot L(A) \cong L(c \cdot A)$.

Last in this section, we give the following technical lemma, which is needed in the proof of Theorem 1.2:

Lemma 6.18. If $A \in \mathrm{sTab}^{\leq}(P)$ is row-equivalent to column-strict, then $\operatorname{word}(c \cdot A)$ can be obtained from word $(A)$ through a series of Knuth equivalences and largersmaller transpositions. In particular, $\operatorname{part}(\operatorname{RS}(A)) \leq \operatorname{part}(\operatorname{RS}(c \cdot A))$.

Proof. Let

$$
\left(a_{1}, \ldots, a_{2 q+1}, b_{1}, \ldots, b_{p}, 0,-b_{p}, \ldots,-b_{1},-a_{2 q+1}, \ldots,-a_{1}\right)=\operatorname{word}(A) .
$$

Due to Theorem 6.13, since

$$
\operatorname{word}\left(A^{L^{-}}\right)=\left(a_{1}, \ldots, a_{q}, b_{1}, \ldots, b_{p},-a_{2 q+1}, \ldots,-a_{q+1}\right),
$$

it suffices to show that

$$
\begin{aligned}
\left(a_{1}, \ldots, a_{q}, b_{1}, \ldots, b_{p},-a_{2 q+1},\right. & \ldots,-a_{q+1}, 0, \\
& \left.a_{q+1}, \ldots, a_{2 q+1},-b_{p}, \ldots,-b_{1},-a_{q}, \ldots,-a_{1}\right)
\end{aligned}
$$

can be obtained from word $(A)$ by a sequence of larger-smaller transpositions and Knuth equivalences. First, we can swap $a_{2 q+1}$ with its right neighbour and $-a_{2 q+1}$ with its left neighbour repeatedly until we get a word with $a_{2 q+1}, 0,-a_{2 q+1}$ in the middle; then, we can swap $a_{2 q+1}$ with 0 , then swap $a_{2 q+1}$ with $-a_{2 q+1}$, then swap 
0 with $-a_{2 q+1}$ so that we have $-a_{2 q+1}, 0, a_{2 q+1}$ in the middle of our word. Now we can repeat this process with $a_{2 q}$ and $a_{2 q}$, then $a_{2 q-1}$ and $-a_{2 q-1}$, and so on. Eventually, since $a_{q+1}>0$, we will get

$$
\begin{aligned}
& \left(a_{1}, \ldots, a_{q}, b_{1}, \ldots, b_{p},-a_{2 q+1}, \ldots,-a_{q+1}, 0\right. \\
& \left.a_{q+1}, \ldots, a_{2 q+1},-b_{p}, \ldots,-b_{1},-a_{q}, \ldots,-a_{1}\right) .
\end{aligned}
$$

\section{The general case}

Now we return to the case of general $\boldsymbol{p}$ as in (3.1). As usual, $P$ is the symmetric pyramid of $\boldsymbol{p}$, with rows labelled $1, \ldots, r, 0,-r, \ldots,-1$ from top to bottom.

7A. The component group action. In this section, we describe the action of the component group $C$ on the subset of $\mathrm{sTab}^{\leq}(P)$ corresponding to finite-dimensional $U(\mathfrak{g}, e)$-modules. The discussion here is completely analogous to the situation for even multiplicity nilpotent elements as described in [Brown and Goodwin 2013c, $\S 5.5]$, so we are quite brief. We use the notation for the component group $C$ from Section 3D.

The operation of $c$ has been defined on three row s-tables in Section 5 and Section 6 , and this can be extended to any s-table by just acting on the middle three rows. To define the action of the $c_{k}$, we proceed in exact analogy with [Brown and Goodwin 2013c, §5.5]. That is, we use row swapping operations $\bar{s}_{i} \star$ to move row $i_{k}$ to row $r$, then we apply $c$, and then we apply the reverse row swaps. So for $A \in \operatorname{sTab}^{\leq}(P)$ and $\tau=\bar{s}_{i_{k}} \bar{s}_{i_{k}+1} \ldots \bar{s}_{r-1} \in \bar{S}_{r}$ we have

$$
c_{k} \cdot A=\tau^{-1} \star(c \cdot(\tau \star A)) .
$$

Of course, this will not be defined for all $A \in \operatorname{sTab}^{\leq}(P)$, but the following proposition can be proved in the same way as Proposition 5.5 of the same reference, and we require Proposition 4.6 for the proof:

Proposition 7.1. Let $A \in \mathrm{sTab}^{\leq}(P)$, and suppose that $L(A)$ is finite-dimensional. Then $c_{k} \cdot A$ is defined and $L\left(c_{k} \cdot A\right) \cong c_{k} \cdot L(A)$.

7B. Proof of main theorem. Now we are in a position to prove Theorem 1.2:

Proof of Theorem 1.2. The statement in the theorem about the component group action is given by Proposition 7.1.

Suppose that $A$ is justified row-equivalent to column-strict. Then $L(A)$ is finitedimensional by Theorems 4.4, 4.8 and 2.4, and thus $b \cdot L(A)$ is finite-dimensional for any $b \in C$ by Proposition 7.1.

We are left to prove that if $L(A)$ is finite-dimensional, then $A \in \operatorname{sTab}^{c}(P)$. We prove this by induction on $r$. The case $r=0$ is trivial, and the case $r=1$ is given by Lemmas 5.1 and 6.1 and Theorems 5.4 and 6.17 . 
Now, assume that $L(A)$ is finite-dimensional and $r \geq 2$. Using an inductive argument based on "Levi subalgebras" of $U(\mathfrak{g}, e)$, just as in the proof of [Brown and Goodwin 2013a, Theorem 5.13], we may assume that $A_{-2}^{2}$ is justified rowequivalent to column-strict, where $A_{-2}^{2}$ denotes the s-table obtained from $A$ by removing rows 1 and -1 . Also by Lemma 4.7 we have that $A_{r}^{1}$ is justified rowequivalent to column-strict, where $A_{r}^{1}$ is the table formed by rows 1 to $r$ of $A$.

Therefore, we can permute entries in the left justification of $A_{-2}^{2}$ so that all the columns are strictly decreasing. Furthermore, we can place each of the entries in row 1 of $A$ over a column so each entry is larger than the entry immediately below it. Then we can place each of the entries of row -1 of $A$ under a column in the left justification of $A_{-2}^{2}$ so that each entry is smaller than the entry above it, and we can do this skew-symmetrically in the sense that if $a$ is an entry in row 1 of $A$ and $a$ is placed over a column whose top entry is $b$, then we can place $-a$ under a column whose bottom entry is $-b$. Let $A_{l}$ denote the resulting diagram.

Let $\boldsymbol{q}=\operatorname{part}(\mathrm{RS}(A))$. As explained below, the conditions above along with Theorem 4.8 give restrictions on the possibilities for $\boldsymbol{q}$. The proof is completed with combinatorial arguments that show that either $\boldsymbol{q}=\boldsymbol{p}$ or $i_{1}=1$, and that $\operatorname{part}\left(\operatorname{RS}\left(c_{1} \cdot A\right)\right)=\boldsymbol{p}$. So by Theorem 4.4 , either $A$ or $c_{1} \cdot A$ is row-equivalent to column-strict.

In the diagram $A_{l}$, let $x$ be the number of columns that go through all the rows, let $y$ be the number of columns that go through all the rows except the top row (so $y$ is also the number of columns that go through all the rows except the bottom row), and let $z$ be the number of columns that go through all the rows except the top and bottom row. Further, let $u$ be the number of columns that go through all the rows except the middle row, let $v$ be the number of columns that go through all the rows except the top row and the middle row (so $v$ is also the number of columns that go through all the rows except the middle row and the bottom row), and let $w$ be the number of columns that go through all the rows except the top, middle and bottom rows. Note that $x+y+u+v=p_{1}$ and $x+2 y+z+u+2 v+w=p_{2}$. So we have $x$ strictly decreasing columns of length $2 r+1,2 y+u$ strictly decreasing columns of length $2 r, z+2 v$ strictly decreasing columns of length $2 r-1$, and $w$ strictly decreasing columns of length $2 r-2$.

By counting the lengths of the other columns in $A_{l}$ similarly, and using Lemma 4.2, we can conclude that

$$
\boldsymbol{q}^{T} \geq\left((2 r+1)^{x},(2 r)^{2 y+u},(2 r-1)^{z+2 v},(2 r-2)^{w},(2 r-4)^{p_{3}-p_{2}}, \ldots, 2^{p_{r-1}-p_{r}}\right)
$$

if $p_{0} \leq p_{r-1}$, and

$$
\begin{aligned}
\boldsymbol{q}^{T} \geq & \left((2 r+1)^{x},(2 r)^{2 y},(2 r-1)^{z},(2 r-3)^{p_{3}-p_{2}}, \ldots,(2 r-2 k+5)^{p_{k-1}-p_{k-2}},\right. \\
& \left.(2 r-2 k+3)^{p_{0}-p_{k}-1},(2 r-2 k+2)^{p_{k}-p_{0}},(2 r-2 k)^{p_{k+1}-p_{k}}, \ldots, 2^{p_{r-1}-p_{r}}\right)
\end{aligned}
$$


if $p_{0}>p_{r-1}$, where $k$ is the number such that $p_{i} \geq p_{0}$ if and only if $i \geq k$ (note that in this case we have $u=v=w=0$ ).

Thus we get that

$$
\boldsymbol{q} \leq\left(p_{r}^{2}, p_{r-1}^{2}, \ldots, p_{2}^{2}, p_{2}-w, x+2 y+u, x\right) \quad \text { if } p_{0} \leq p_{r-1}
$$

and

$$
\boldsymbol{q} \leq\left(p_{r}^{2}, p_{r-1}^{2}, \ldots, p_{k-1}^{2}, p_{0}, p_{k}^{2}, \ldots, p_{2}^{2}, p_{1}-z, x\right) \quad \text { if } p_{0}>p_{r-1} .
$$

Since we also have $\boldsymbol{p}$ as a lower bound of $\boldsymbol{q}$, this implies that

$$
\boldsymbol{q}=\left(p_{r}^{2}, p_{r-1}^{2}, \ldots, p_{2}^{2}, a, b, c\right) \quad \text { if } p_{0} \leq p_{r-1}
$$

and

$$
\boldsymbol{q}=\left(p_{r}^{2}, p_{r-1}^{2}, \ldots, p_{k}^{2}, p_{0}, p_{k-1}^{2}, \ldots, p_{2}^{2}, a, b\right) \quad \text { if } p_{0}>p_{r-1},
$$

for positive integers $a, b, c$. Since $\operatorname{content}(\boldsymbol{q})=\operatorname{content}(\boldsymbol{p})$, we get a very limited number of possibilities for $a, b, c$, as explained below.

From now we restrict to the case $\mathfrak{g}=\mathfrak{s p}_{2 n}$ in this proof, as the case $\mathfrak{g}=\mathfrak{s o}_{2 n+1}$ is entirely similar; in some places we would require references from Section 6 rather than Section 5.

We know that $p_{0}$ must be even. If $p_{0}<p_{1}$ and $p_{1}$ is even, then we must have $(a, b, c)=\left(p_{1}, p_{1}, p_{0}\right)$ or $(a, b, c)=\left(p_{1}+1, p_{1}-1, p_{0}\right)$. If $p_{0}<p_{1}$ and $p_{1}$ is odd, then $(a, b, c)=\left(p_{1}, p_{1}, p_{0}\right)$. If $p_{1}<p_{0}<p_{2}$, then $p_{1}$ is even and $(a, b, c)=\left(p_{0}, p_{1}, p_{1}\right)$. Finally, if $p_{0}>p_{2}$, then $p_{1}$ is even and $(b, c)=\left(p_{1}, p_{1}\right)$.

By Theorem 4.4, if $\boldsymbol{q}=\boldsymbol{p}$, then $A$ is justified row-equivalent to column-strict, and we are done. So for the rest of this proof we will assume that $\boldsymbol{q} \neq \boldsymbol{p}$; so, we are assuming that $p_{0}<p_{r}, p_{r}$ is even, and

$$
\boldsymbol{q}=\left(p_{r}^{2}, \ldots, p_{2}^{2}, p_{1}+1, p_{1}-1, p_{0}\right) .
$$

It is be useful to record that

$$
\begin{aligned}
\boldsymbol{q}^{T}=\left((2 r+1)^{p_{0}},(2 r)^{p_{1}-p_{0}-1},(2 r-1)^{2},\right. & \\
& \left.(2 r-2)^{p_{2}-p_{1}-1},(2 r-4)^{p_{3}-p_{2}}, \ldots, 2^{p_{r-1}-p_{r}}\right) .
\end{aligned}
$$

Let $\sigma=s_{r-1} \ldots \bar{s}_{2} \bar{s}_{1}$ and $A^{\prime}=\sigma \star A$; then $\operatorname{RS}\left(A^{\prime}\right)=\operatorname{RS}(A)$ by Proposition 4.6. Then the lengths of the middle three rows of $A^{\prime}$ are given by $p_{1}, p_{0}, p_{1}$. Let $B$ be the middle three rows of $A^{\prime}$.

We claim that $\operatorname{part}(\operatorname{RS}(B))=\left(p_{1}+1, p_{1}-1, p_{0}\right)$. To see this, first we suppose that $\operatorname{part}(\mathrm{RS}(B))=\left(p_{r}, p_{r}, p_{0}\right)$. Then $B$ is justified row-equivalent to columnstrict. Now, since $\left(A^{\prime}\right)_{r}^{1}$ is justified row-equivalent to column-strict, this allows us to find $p_{0}$ disjoint decreasing words of length $2 r+1$ that are disjoint from a further $p_{1}-p_{0}$ disjoint decreasing words of length $2 r$. Thus, by Lemma 4.2, 
$\boldsymbol{q}^{T} \geq\left((2 r+1)^{p_{0}},(2 r)^{p_{1}-p_{0}}, *\right)$, which contradicts (7.3). Now we also cannot have that any part of part $(\operatorname{RS}(B))$ is larger than $p_{r}+1$, since then we could use the fact that all the rows of $A^{\prime}$ are increasing to conclude that $\operatorname{part}(\boldsymbol{q})$ would be strictly larger than a partition of the form

$$
\left(p_{1}^{2}, p_{2}^{2}, \ldots, p_{r-1}^{2}, p_{r}+1, *\right),
$$

which contradicts (7.2).

Now, we have by Theorem 5.4 that $\operatorname{part}(\operatorname{RS}(c \cdot B))=\left(p_{0}, p_{1}, p_{1}\right)$. We also have by Lemma 5.5 that $\operatorname{part}\left(\operatorname{RS}\left(c_{1} \cdot A\right)\right) \leq \operatorname{part}(\operatorname{RS}(A))$.

We need to argue that we can find enough descending chains of maximal or near maximal length in $c \cdot A^{\prime}$ to force $\operatorname{RS}\left(c_{1} \cdot A\right)$ to have shape $\boldsymbol{p}$. We have by Lemma 4.7 that $\left(c \cdot A^{\prime}\right)_{r}^{1}$ is justified row-equivalent to column-strict. Further, by Theorem 4.4 we have that $c \cdot B$ is justified row-equivalent to column-strict.

We can find $p_{0}$ descending strings of length 3 and $p_{1}-p_{0}$ strings of length 2 , and all these strings start in row $r$ and end in row $-r$. Since $\left(c \cdot A^{\prime}\right)_{r}^{1}$ is justified row-equivalent to column-strict, it has $p_{1}$ strings of length $r$ ending in row $r$, and $\left(c \cdot A^{\prime}\right)_{-1}^{-r}$ has $p_{1}$ strings of length $r$ starting in row -1 . So we can glue these strings together along their entries in rows 1 and -1 to obtain $p_{0}$ disjoint decreasing strings of length $2 r+1$ that are disjoint from $p_{1}-p_{0}$ disjoint decreasing strings of length $2 r$. So if $\boldsymbol{q}^{\prime}=\operatorname{part}(\operatorname{RS}(c \cdot A))$, we can conclude that $\boldsymbol{q}^{\prime T} \geq\left((2 r+1)^{p_{0}},(2 r)^{p_{1}-p_{0}}, *\right)$, which implies that $\boldsymbol{q}^{\prime}=\boldsymbol{p}$, so $c_{1} \cdot A$ is justified row-equivalent to column-strict, as required.

Finally, this theorem along with Theorems 2.3 and 4.5 immediately imply the following classification of the primitive ideals with associated variety equal to $\overline{G \cdot e}$ :

Corollary 7.4. The set of primitive ideals with associated variety $\overline{G \cdot e}$ is equal to

$$
\left\{\operatorname{Ann}_{U(\mathfrak{g})} L\left(\lambda_{A}\right) \mid A \in \operatorname{sTab}^{c}(P)\right\} .
$$

\section{Appendix: An alternative version of the Barbasch-Vogan algorithm}

In this appendix, we consider the alternative version of the Barbasch-Vogan algorithm for $\mathfrak{s o}_{2 n+1}$, mentioned in Section 4C above. Our main result is Corollary A.7, which shows that this adapted version gives the same output as the original version. Below, we recall the algorithm, then, in the subsequent subsections construct the machinery required to prove Corollary A.7.

Some terminology and notation used in this section are as follows. By a Young diagram we mean a finite collection of boxes, or cells, arranged in left-justified rows, with the row lengths weakly decreasing. We often identify a Young diagram with its underlying partition. A tableau is a filling of a Young diagram by integers with weakly increasing rows and strictly decreasing columns. We write $\operatorname{part}(T)$ for 
the partition underlying a tableau $T$. The Robinson-Schensted algorithm is denoted by RS.

The algorithms. Let $\boldsymbol{q}=\left(q_{1} \leq q_{2} \leq \cdots \leq q_{m}\right)$ be a partition. By inserting 0 at the beginning if necessary, we may assume that $m$ is odd. Let $\left(s_{1}, \ldots, s_{k}\right),\left(t_{1}, \ldots, t_{l}\right)$ be such that

$$
\left\{q_{1}, q_{2}+1, q_{3}+2, \ldots, q_{r}+r-1\right\}=\left\{2 s_{1}, \ldots, 2 s_{k}, 2 t_{1}+1, \ldots, 2 t_{l}+1\right\}
$$

(as unordered lists). Now we define the content of $\boldsymbol{q}$ to be the unordered list

$$
\operatorname{content}(\boldsymbol{q})=\left\{s_{1}, \ldots, s_{k}, t_{1}, \ldots, t_{l}\right\}
$$

We now state the Barbasch-Vogan algorithm [1982] for the case $\mathfrak{g}=\mathfrak{s o}_{2 n+1}$ in purely combinatorial terms:

\section{Algorithm:}

Input: $\boldsymbol{a}=\left(a_{1}, \ldots, a_{n},-a_{n}, \ldots,-a_{1}\right)$ a skew-symmetric string of integers.

Step 1: Calculate $\boldsymbol{q}=\operatorname{part}\left(\operatorname{RS}\left(a_{1}, \ldots, a_{n},-a_{n}, \ldots,-a_{1}\right)\right)$.

Step 2: Calculate content $(\boldsymbol{q})$.

Let $\left(u_{1} \leq \cdots \leq u_{2 k+1}\right)$ be the sorted list with the same entries as content $(\boldsymbol{q})$.

For $i=1, \ldots, k+1$ let $s_{i}=u_{2 i-1}$.

For $i=1, \ldots, k$ let $t_{i}=u_{2 i}$.

Step 3: Form the list $\left(2 s_{1}+1, \ldots, 2 s_{k+1}+1,2 t_{1}, \ldots, 2 t_{k}\right)$.

In either case, let $\left(v_{1}<\cdots<v_{k}\right)$ be this list after sorting.

Output: $\mathrm{BV}(\boldsymbol{a})=\boldsymbol{q}^{\prime}=\left(v_{1}, v_{2}-1, \ldots, v_{2 k+1}-2 k\right)$.

The modified version is denoted by $\mathrm{BV}^{\prime}$ and works in exactly the same way as $\mathrm{BV}$, except that in Step 1 it calculates $\operatorname{RS}\left(a_{1}, \ldots, a_{n}, 0,-a_{n}, \ldots,-a_{1}\right)$ instead of $\operatorname{RS}\left(a_{1}, \ldots, a_{n},-a_{n}, \ldots,-a_{1}\right)$.

Domino tableaux. We require some facts about domino tableaux, which we collate below.

There are two types of domino tableaux: those with an even number of boxes and those with an odd number of boxes. A domino tableau with an even number of boxes is a Young diagram that has been tiled with $2 \times 1$ and $1 \times 2$ dominoes, where each domino is labelled with a positive integer, such that the rows are increasing and the columns are decreasing. A domino tableau with an odd number of boxes is the same as a domino tableau with an even number of boxes, except it also has a $1 \times 1$ box labelled with 0 , which must necessarily occur in the lower-left position. 
For example,
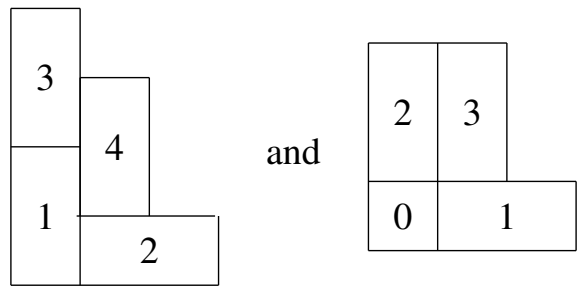

are domino tableaux.

Given a domino tableau $R$, we let $\operatorname{part}(R)$ denote the partition underlying $R$, i.e., the partition given by the row lengths of $R$. We say that a partition has domino shape if it is the underlying partition of a domino tableau.

The following lemma is straightforward to prove by induction:

Lemma A.1. Let $\boldsymbol{p}=\left(p_{1} \leq p_{2} \leq \cdots \leq p_{m}\right)$ be a partition, where $p_{1}$ may be 0 and $m$ is odd. Choose $r_{1}, \ldots, r_{k}$ and $s_{1}, \ldots, s_{l}$ so that

$$
\left\{p_{1}, p_{2}+1, \ldots, p_{m}+m-1\right\}=\left\{2 r_{1}, \ldots, 2 r_{k}, 2 s_{1}+1, \ldots, 2 s_{l}+1\right\}
$$

(as unordered lists). If $\boldsymbol{p}$ has domino shape and has an even number of boxes, then $k=l+1$. If $\boldsymbol{p}$ has domino shape and has an odd number of boxes, then $k+1=l$.

Let $T$ be a tableau whose boxes are labelled by the integers $-n, \ldots,-1,1, \ldots, n$ or the integers $-n, \ldots,-1,0,1, \ldots, n$. We recall an algorithm DT, which takes as input such a tableau and outputs a domino tableau; it was defined in [Barbasch and Vogan 1982]. To define DT( $T)$, first note that $-n$ must occur in the lower-left corner of $T$. Swap $-n$ with the smaller of its neighbours that lie above or to the right of $-n$. Continue swapping $-n$ with its smaller neighbour that is either above or right of it. If the last number that $-n$ is swapped with is not $n$ then we say that $\mathrm{DT}(T)$ is undefined. Otherwise, replace the squares with $-n$ and $n$ with a domino containing $n$. Now repeat this procedure for $1-n, 2-n, \ldots,-1$, treating any squares that have been replaced with dominoes as if they were not present. If for any $i$ the last number that $-i$ is replaced with is not $i$ then $\operatorname{DT}(T)$ is undefined. Otherwise, we eventually get a domino tableau.

For example, suppose

$$
T=\operatorname{RS}(-2,-3,1,0,-1,3,2)=\begin{array}{rr|r|r|}
\hline 1 & \multicolumn{2}{|c|}{} \\
\hline-2 & 0 & 3 \\
\hline-3 & -1 & 2 \\
\hline
\end{array}
$$

Now, when we apply the above algorithm we first swap -3 with -2 , then with 0 , then with 3 . Now, replace the boxes containing 3 and -3 with a domino containing 3 . 
This results in the following diagram:

\begin{tabular}{|c|c|c|}
\hline 1 & \multicolumn{2}{|c}{} \\
\hline 0 & \multicolumn{2}{|c|}{3} \\
\hline-2 & -1 & 2 \\
\hline
\end{tabular}

Now, -2 first swaps with -1 , then 2 , which results in the following diagram:

\begin{tabular}{|c|c|}
\hline 1 & \\
\hline 0 & 3 \\
\hline-1 & 2 \\
\hline
\end{tabular}

Finally, -1 swaps with 0 , then 1 , and the resulting domino tableau is

$$
\mathrm{DT}(T)=\begin{array}{|l|l|}
\hline 1 & \multicolumn{2}{|l}{} \\
\cline { 3 - 3 } & 3 \\
\hline 0 & 2 \\
\hline
\end{array}
$$

Let $W_{n}$ denote the Weyl group of type $B_{n}$ acting on $\{ \pm 1, \ldots, \pm n\}$ in the natural way. Then the image of $(-n, \ldots,-1,1, \ldots, n)$ under the action of some $\sigma \in W_{n}$ is called a signed permutation of $(-n, \ldots,-1,1, \ldots, n)$. A signed permutation of $(-n, \ldots,-1,0,1, \ldots, n)$ is defined similarly.

The next lemma follows from Proposition 2.3.3 and Theorem 4.1.1 in [van Leeuwen 1996]:

Lemma A.3. Let $\boldsymbol{a}=\left(a_{1}, \ldots, a_{n},-a_{n}, \ldots,-a_{1}\right)$ be a signed permutation of $(-n, \ldots,-1,1, \ldots, n)$ and $\boldsymbol{b}=\left(b_{1}, \ldots, b_{n}, 0,-b_{n}, \ldots,-b_{1}\right)$ a signed permutation of $(-n, \ldots,-1,0,1, \ldots, n)$. Then $\mathrm{DT}(\mathrm{RS}(\boldsymbol{a}))$ and $\mathrm{DT}(\mathrm{RS}(\boldsymbol{b}))$ are defined.

We may identify $W_{n}$ with the signed permutations of $(-n, \ldots,-1,1, \ldots, n)$ or the signed permutations of $(-n, \ldots,-1,0,1, \ldots, n)$. Under this identification, we consider the algorithms defined in [Garfinkle 1990, §2] to map a signed permutation of $(-n, \ldots,-1,1, \ldots, n)$ or $(-n, \ldots,-1,0,1, \ldots, n)$ to a domino tableau. We denote these versions of Garfinkle's algorithm by $\mathrm{G}_{0}$ and $\mathrm{G}_{1}$ respectively.

Proposition A.4 [van Leeuwen 1996, Proposition 4.2.3].

(i) If $w$ is a signed permutation of $(-n, \ldots,-1,1, \ldots, n)$, then $\operatorname{DT}(\operatorname{RS}(w))=$ $\mathrm{G}_{0}(w)$.

(ii) If $w$ is a signed permutation of $(-n, \ldots,-1,0,1, \ldots, n)$, then $\operatorname{DT}(\operatorname{RS}(w))=$ $\mathrm{G}_{1}(w)$. 
Our aim is to show that $\operatorname{part}\left(\operatorname{RS}\left(a_{1}, \ldots, a_{n},-a_{n}, \ldots,-a_{1}\right)\right)$ has the same content as $\operatorname{part}\left(\operatorname{RS}\left(a_{1}, \ldots, a_{n}, 0,-a_{n}, \ldots,-a_{1}\right)\right)$. We do this by exploiting the results in [Pietraho 2010], which explain how to relate $\mathrm{G}_{0}\left(a_{1}, \ldots, a_{n},-a_{n}, \ldots,-a_{1}\right)$ and $\mathrm{G}_{1}\left(a_{1}, \ldots, a_{n}, 0,-a_{n}, \ldots,-a_{1}\right)$. To explain these results, we need to define the cycles of a standard domino tableau. This requires a few other definitions as well.

We define coordinates on a Young diagram by labelling its rows and columns. We declare that the bottom row is row 1 , the row above the bottom is row 2 , and so on. We declare that the left most column is column 1 , the column to its right is column 2 , and so on. Now we say the box in position $(i, j)$ is fixed if $i+j$ is odd and the diagram has an even number of boxes or if $i+j$ is even and the diagram has an odd number of boxes.

Let $R$ be a domino tableau, and let $D(k)$ be a domino with label $k$ in $R$. If the fixed coordinate of $D(k)$ occurs in the lower box or right box of $D(k)$, let $E$ denote the square below and to the right of the fixed coordinate of $D(k)$. If the fixed coordinate of $D(k)$ occurs in the upper box or left box of $D(k)$, let $E$ denote the square above and to the left of the fixed coordinate of $D(k)$. We label $E$ with the integer $m$ determined via

$$
m=\left\{\begin{aligned}
l & \text { if } E \text { is a square in } R \text { and } l \text { is the label of } E \text { 's square in } R, \\
-1 & \text { if either coordinate of } E \text { is } 0, \\
\infty & \text { if } E \text { lies above or to the right of } R .
\end{aligned}\right.
$$

Now, we define $D^{\prime}(k)$ to be a domino containing two squares, one in the fixed position of $D(k)$ and the other adjacent to $E$ and such that the subdiagram containing $D^{\prime}(k)$ and $E$ has decreasing columns and increasing rows.

For example, if

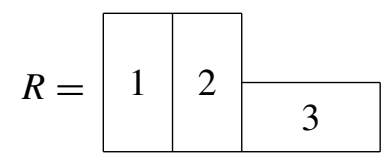

then $D^{\prime}(1)$ is a domino occupying positions $(2,1)$ and $(3,1), D^{\prime}(2)$ is a domino occupying positions $(1,2)$ and $(1,3)$, and $D^{\prime}(3)$ is a domino occupying positions $(1,4)$ and $(1,5)$.

Suppose a domino tableau is labelled with $\{1, \ldots, n\}$. We use this to generate an equivalence relation on $\{1, \ldots, n\}$ via $i \sim j$ if $D(j)$ and $D^{\prime}(i)$ share a box. The cycles of a domino tableau are the equivalence classes of this equivalence relation. For example, if $R$ is as above, then the cycles of $R$ are $\{1\}$ and $\{2,3\}$.

If $R$ is a domino tableau with an even number of boxes and $c$ is a cycle of $R$, then we can define a new domino tableau $R^{\prime}=\operatorname{MT}(R, c)$ by replacing $D(k)$ with $D^{\prime}(k)$ for every $k \in c$. This will remove one box and add one box to the underlying Young diagram of $R$. If the box removed is in position $(1,1)$, then we put a box 
with 0 in position $(1,1)$ of $R^{\prime}$, so that we do in fact get another domino tableau. For example, if $R$ is as above, then

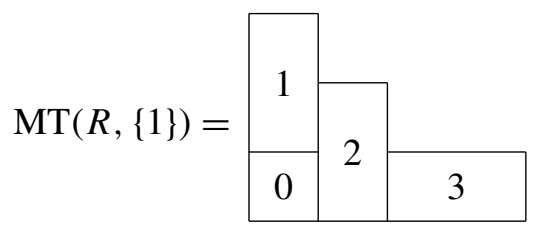

and

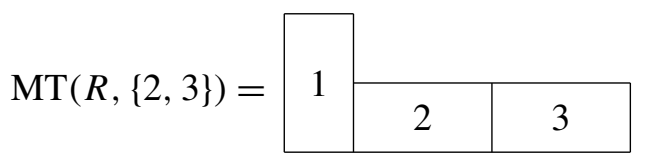

Observe that the operator MT removes a box and adds a box to the Young diagram underlying $R$, and that the removed box is either in position $(1,1)$, or is a removable box of $R$; that is, if it is removed, you still have a valid Young diagram.

A key feature of MT is that is does not change the content of the underlying partition:

Theorem A.5. Let $R$ be a domino tableau with an even number of boxes, let $c$ be a cycle of $R$, and let $\boldsymbol{p}=\operatorname{part}(R)$ and $\boldsymbol{q}=\operatorname{part}(\mathrm{MT}(R, c))$. Then content $(\boldsymbol{p})=$ $\operatorname{content}(\boldsymbol{q})$.

Proof. First we rule out the case that $\boldsymbol{p}$ has an odd number (say, $2 m+1$ ) of parts and $\boldsymbol{q}$ has one more part than $\boldsymbol{p}$. Suppose for a contradiction that $\boldsymbol{q}$ has $2 m+2$ parts, so the top row of $\operatorname{MT}(R, c)$ has one box. Let $D^{\prime}(k)$ be the domino in $\operatorname{MT}(S, c)$ that covers this box. So the box in the fixed position of $D^{\prime}(k)$ must be the box in position $(2 m+1,1)$, which is a contradiction since $2 m+1+1$ is even.

Next we rule out the case that $\boldsymbol{p}$ has an even number (say, $2 m$ ) of parts and $\boldsymbol{q}$ has one less part than $\boldsymbol{p}$. Suppose this is the case, so the top row $\boldsymbol{p}$ has length one, so there must be a domino $D(k)$ which occupies positions $(2 m-1,1)$ and $(2 m, 1)$ of $\boldsymbol{p}$. Now, $(2 m, 1)$ is the fixed position of this domino, so $D^{\prime}(k)$ will also have a box in position $(2 m, 1)$; hence, $\boldsymbol{q}$ has at least $2 m$ parts, which is a contradiction. Thus we have established that the number of integers in $\operatorname{content}(\boldsymbol{q})$ is the same as the number of integers in content $(\boldsymbol{p})$.

Let $\boldsymbol{p}=\left(p_{1} \leq \cdots \leq p_{2 m+1}\right)$, where $p_{1}$ may be 0 . Now we consider the case that $\operatorname{MT}(R, c)$ has the same number of boxes as $R$. Let $\boldsymbol{q}=\left(q_{1} \leq \cdots \leq q_{2 m+1}\right)$, where $q_{1}$ may be 0 . So we must have that $q_{i}=p_{i}$ except for $i=j$ and $i=k$ for some integers $j, k$ where $j \neq k$, and $q_{j}=p_{j}+1$ and $q_{k}=p_{k}-1$. By Lemma A.1, we have that one of $p_{j}+j-1, p_{k}+k-1$ must be even and one must be odd, because otherwise $\left(q_{1}, q_{2}+1, \ldots, q_{2 m+1}+2 m\right)$ would not have one more even element than odd elements. The box at position $\left(j, p_{j}\right)$ of $\mathrm{MT}(R, c)$ is the box that gets 
added to the Young diagram of $R$. Thus, this box is a box that is in $D^{\prime}(k)$ but not in $D(k)$. This implies that this box is not the box in fixed position in $D^{\prime}(k)$; thus, $p_{j}+j$ is even, so $p_{j}+j-1$ must be odd, and $p_{k}+k-1$ is even. This implies that $\boldsymbol{p}$ and $\boldsymbol{q}$ have the same content.

Now we consider the case that $\mathrm{MT}(R, c)$ has one more box than $R$. Let $\boldsymbol{q}=$ $\left(q_{1} \leq \cdots \leq q_{2 m+1}\right)$, where $q_{1}$ may be 0 . So we must have that $q_{i}=p_{i}$ except for $i=j$ for some integer $j$, where $q_{j}=p_{j}+1$. Note that $p_{j}+j-1$ must be even since $\left(q_{1}, q_{2}+1, \ldots, q_{2 m+1}+2 m\right)$ must have one more odd number than even number. This implies that $\boldsymbol{p}$ and $\boldsymbol{q}$ have the same content.

For a list of cycles $c_{1}, \ldots, c_{m}$ of a domino tableau $R$ with an even number of boxes, let $R_{i}=\operatorname{MT}\left(R_{i-1}, c_{i}\right)$, where $R_{0}=R$. Now let $\mathrm{MT}\left(R, c_{1}, \ldots, c_{m}\right)=R_{m}$.

The following theorem is a less specific version of [Pietraho 2010, Theorem 3.1]:

Theorem A.6. Let

$$
R=\mathrm{G}_{0}\left(a_{1}, \ldots, a_{n},-a_{n}, \ldots,-a_{1}\right)
$$

and

$$
R^{\prime}=\mathrm{G}_{1}\left(a_{1}, \ldots, a_{n}, 0,-a_{n}, \ldots,-a_{1}\right),
$$

where $\left(a_{1}, \ldots, a_{n},-a_{n}, \ldots,-a_{1}\right)$ is a signed permutation of $(-n, \ldots,-1,1, \ldots, n)$. Then there exist cycles $c_{1}, \ldots, c_{m}$ of $R$ such that $R^{\prime}=\operatorname{MT}\left(R, c_{1}, \ldots, c_{m}\right)$.

Now we get the following corollary:

Corollary A.7. Let $\boldsymbol{a}=\left(a_{1}, \ldots, a_{n},-a_{n}, \ldots,-a_{1}\right)$ be a skew-symmetric string of integers. Then $\mathrm{BV}(\boldsymbol{a})=\mathrm{BV}^{\prime}(\boldsymbol{a})$.

Proof. This follows from Proposition A.4 and Theorems A.5 and A.6 when $\boldsymbol{a}$ is a signed permutation of $(-n, \ldots,-1,1, \ldots, n)$. The general case follows because $\boldsymbol{q}=\operatorname{RS}\left(a_{1}, \ldots, a_{n},-a_{n}, \ldots,-a_{1}\right)$ and $\boldsymbol{q}^{\prime}=\operatorname{RS}\left(a_{1}, \ldots, a_{n}, 0,-a_{n}, \ldots,-a_{1}\right)$ depend only on the relative order of the $a_{i}$, so we may replace $\boldsymbol{a}$ by a signed permutation without altering $\boldsymbol{q}$ or $\boldsymbol{q}^{\prime}$.

\section{Acknowledgements}

This research is funded by EPSRC grant EP/G020809/1.

\section{References}

[Barbasch and Vogan 1982] D. Barbasch and D. Vogan, "Primitive ideals and orbital integrals in complex classical groups”, Math. Ann. 259:2 (1982), 153-199. MR 83m:22026 Zbl 0489.22010

[Barbasch and Vogan 1983] D. Barbasch and D. Vogan, "Primitive ideals and orbital integrals in complex exceptional groups", J. Algebra 80:2 (1983), 350-382. MR 84h:22038 Zbl 0513.22009

[Brown 2011] J. S. Brown, "Representation theory of rectangular finite W-algebras", J. Algebra 340 (2011), 114-150. MR 2813566 Zbl 06005449 
[Brown and Goodwin 2013a] J. S. Brown and S. M. Goodwin, "Finite dimensional irreducible representations of finite $W$-algebras associated to even multiplicity nilpotent orbits in classical Lie algebras”, Math. Z. 273:1-2 (2013), 123-160. MR 3010154 Zbl 1266.17003

[Brown and Goodwin 2013b] J. S. Brown and S. M. Goodwin, "Non-integral representation theory of even multiplicity finite $W$-algebras", J. Algebra 380 (2013), 30-45. MR 3023226 Zbl 06220586 arXiv 1112.6293

[Brown and Goodwin 2013c] J. S. Brown and S. M. Goodwin, "On changing highest weight theories for finite $W$-algebras”, J. Algebraic Combin. 37:1 (2013), 87-116. MR 3016303 Zbl 06138614

[Brundan et al. 2008] J. Brundan, S. M. Goodwin, and A. Kleshchev, "Highest weight theory for finite W-algebras”, Int. Math. Res. Not. 2008:15 (2008), Art. ID \#rnn051. MR 2009f:17011 Zbl 1211.17024

[Collingwood and McGovern 1993] D. H. Collingwood and W. M. McGovern, Nilpotent orbits in semisimple Lie algebras, Van Nostrand Reinhold, New York, 1993. MR 94j:17001 Zbl 0972.17008

[Fulton 1997] W. Fulton, Young tableaux: with applications to representation theory and geometry, London Mathematical Society Student Texts 35, Cambridge University Press, 1997. MR 99f:05119 Zbl 0878.14034

[Garfinkle 1990] D. Garfinkle, "On the classification of primitive ideals for complex classical Lie algebras, I”, Compositio Math. 75:2 (1990), 135-169. MR 91m:17011 Zbl 0737.17003

[Garfinkle 1993] D. Garfinkle, "On the classification of primitive ideals for complex classical Lie algebras, III”, Compositio Math. 88:2 (1993), 187-234. MR 94i:17017 Zbl 0798.17007

[Jantzen 1983] J. C. Jantzen, Einhüllende Algebren halbeinfacher Lie-Algebren, Ergebnisse der Mathematik und ihrer Grenzgebiete (3) 3, Springer, Berlin, 1983. MR 86c:17011 Zbl 0541.17001

[Jantzen 2004] J. C. Jantzen, "Nilpotent orbits in representation theory", pp. 1-211 in Lie theory, edited by J.-P. Anker and B. Orsted, Progr. Math. 228, Birkhäuser, Boston, 2004. MR 2005c:14055 Zbl 1169.14319

[Joseph 1977] A. Joseph, "A characteristic variety for the primitive spectrum of a semisimple Lie algebra", pp. 102-118 in Non-commutative harmonic analysis (Marseille-Luminy, 1976), edited by J. Carmona and M. Vergne, Lecture Notes in Math. 587, Springer, Berlin, 1977. MR 56 \#8645 Zbl 0374.17004

[Kostant 1978] B. Kostant, "On Whittaker vectors and representation theory”, Invent. Math. 48:2 (1978), 101-184. MR 80b:22020 Zbl 0405.22013

[van Leeuwen 1996] M. A. A. van Leeuwen, "The Robinson-Schensted and Schützenberger algorithms: an elementary approach", Electron. J. Combin. 3:2 (1996), 391-422. Research Paper 15. MR 97e:05200 Zbl 0852.05080

[Losev 2010] I. Losev, "Quantized symplectic actions and W-algebras", J. Amer. Math. Soc. 23:1 (2010), 35-59. MR 2010k:17018 Zbl 1246.17015

[Losev 2011a] I. Losev, "Finite-dimensional representations of W-algebras", Duke Math. J. 159:1 (2011), 99-143. MR 2817650 Zbl 1235.17007

[Losev 2011b] I. Losev, "Finite W-algebras", pp. 1281-1307 in Proceedings of the International Congress of Mathematicians (Hyderabad, 2010), vol. III, edited by R. Bhatia et al., Hindustan Book Agency, New Delhi, 2011. MR 2012g:16001 Zbl 1232.17024

[Losev 2012] I. Losev, "On the structure of the category O for $W$-algebras", pp. 351-368 in Geometric Methods in Representation Theory, II, edited by M. Brion, Séminaires et Congrès 24-II, Société Mathématique de France, Paris, 2012. Zbl 1275.14002

[Losev and Ostrik 2013] I. Losev and V. Ostrik, "Classification of finite dimensional irreducible modules over W-algebras", preprint, 2013. To appear in Compositio Mathematica. arXiv 1202.6097 
[Lusztig 1979] G. Lusztig, "A class of irreducible representations of a Weyl group", Nederl. Akad. Wetensch. Indag. Math. 82:3 (1979), 323-335. MR 81a:20052 Zbl 0435.20021

[Miličić and Soergel 1997] D. Miličić and W. Soergel, "The composition series of modules induced from Whittaker modules", Comment. Math. Helv. 72:4 (1997), 503-520. MR 99e:17010 Zbl 0956.17004

[Pietraho 2010] T. Pietraho, "A relation for domino Robinson-Schensted algorithms", Ann. Comb. 13:4 (2010), 519-532. MR 2011e:05280 Zbl 1229.05283

[Premet 2007] A. Premet, "Enveloping algebras of Slodowy slices and the Joseph ideal", J. Eur. Math. Soc. (JEMS) 9:3 (2007), 487-543. MR 2008c:17006 Zbl 1134.17307

[Skryabin 2002] S. Skryabin, "A category equivalence", appendix (pp. 50-53) to A. Premet's "Special transverse slices and their enveloping algebras", Adv. Math. 170:1 (2002), 1-55.

Received November 22, 2012. Revised October 15, 2013.

JONATHAN BROWN

Department of Mathematics, Computer Science, and Statistics

SUNY ONEONTA

ONEONTA, NY 13820

UNITED STATES

Jonathan.Brown@oneonta.edu

SIMON M. GoOdWIN

SCHOOL OF MATHEMATICS

UNIVERSITY OF BIRMINGHAM

EDGBASTON

BIRMINGHAM

B15 2TT

UNITED KINGDOM

s.m.goodwin@bham.ac.uk 


\title{
PACIFIC JOURNAL OF MATHEMATICS
}

\author{
msp.org/pjm
}

Founded in 1951 by E. F. Beckenbach (1906-1982) and F. Wolf (1904-1989)

\section{EDITORS}

Don Blasius (Managing Editor)

Department of Mathematics

University of California

Los Angeles, CA 90095-1555

blasius@math.ucla.edu

\author{
Paul Balmer \\ Department of Mathematics \\ University of California \\ Los Angeles, CA 90095-1555 \\ balmer@math.ucla.edu \\ Robert Finn \\ Department of Mathematics \\ Stanford University \\ Stanford, CA 94305-2125 \\ finn@math.stanford.edu \\ Sorin Popa \\ Department of Mathematics \\ University of California \\ Los Angeles, CA 90095-1555 \\ popa@math.ucla.edu
}

\author{
Vyjayanthi Chari \\ Department of Mathematics \\ University of California \\ Riverside, CA 92521-0135 \\ chari@math.ucr.edu \\ Kefeng Liu \\ Department of Mathematics \\ University of California \\ Los Angeles, CA 90095-1555 \\ liu@math.ucla.edu \\ Jie Qing \\ Department of Mathematics \\ University of California \\ Santa Cruz, CA 95064 \\ qing@ cats.ucsc.edu
}

\section{PRODUCTION}

Silvio Levy, Scientific Editor, production@msp.org

\section{SUPPORTING INSTITUTIONS}

ACADEMIA SINICA, TAIPEI

CALIFORNIA INST. OF TECHNOLOGY

INST. DE MATEMÁTICA PURA E APLICADA

KEIO UNIVERSITY

MATH. SCIENCES RESEARCH INSTITUTE

NEW MEXICO STATE UNIV.

OREGON STATE UNIV.

\author{
STANFORD UNIVERSITY \\ UNIV. OF BRITISH COLUMBIA \\ UNIV. OF CALIFORNIA, BERKELEY \\ UNIV. OF CALIFORNIA, DAVIS \\ UNIV. OF CALIFORNIA, LOS ANGELES \\ UNIV. OF CALIFORNIA, RIVERSIDE \\ UNIV. OF CALIFORNIA, SAN DIEGO \\ UNIV. OF CALIF., SANTA BARBARA
}

\author{
Daryl Cooper \\ Department of Mathematics \\ University of California \\ Santa Barbara, CA 93106-3080 \\ cooper@math.ucsb.edu \\ Jiang-Hua Lu \\ Department of Mathematics \\ The University of Hong Kong \\ Pokfulam Rd., Hong Kong \\ jhlu@maths.hku.hk \\ Paul Yang \\ Department of Mathematics \\ Princeton University \\ Princeton NJ 08544-1000 \\ yang@math.princeton.edu
}

These supporting institutions contribute to the cost of publication of this Journal, but they are not owners or publishers and have no responsibility for its contents or policies.

See inside back cover or msp.org/pjm for submission instructions.

The subscription price for 2014 is US $\$ 410 /$ year for the electronic version, and \$535/year for print and electronic.

Subscriptions, requests for back issues and changes of subscribers address should be sent to Pacific Journal of Mathematics, P.O. Box 4163, Berkeley, CA 94704-0163, U.S.A. The Pacific Journal of Mathematics is indexed by Mathematical Reviews, Zentralblatt MATH, PASCAL CNRS Index, Referativnyi Zhurnal, Current Mathematical Publications and Web of Knowledge (Science Citation Index).

The Pacific Journal of Mathematics (ISSN 0030-8730) at the University of California, c/o Department of Mathematics, 798 Evans Hall \#3840, Berkeley, CA 94720-3840, is published twelve times a year. Periodical rate postage paid at Berkeley, CA 94704, and additional mailing offices. POSTMASTER: send address changes to Pacific Journal of Mathematics, P.O. Box 4163, Berkeley, CA 94704-0163.

PJM peer review and production are managed by EditFLOW ${ }^{\circledR}$ from Mathematical Sciences Publishers.

\section{PUBLISHED BY}

\section{mathematical sciences publishers \\ nonprofit scientific publishing}

http://msp.org/

(C) 2014 Mathematical Sciences Publishers 


\section{PACIFIC JOURNAL OF MATHEMATICS}

Volume 269 No. $1 \quad$ May 2014

The asymptotic behavior of Palais-Smale sequences on manifolds with $\quad 1$ boundary

SÉRGIO AlMARAZ

The cup subalgebra of a $\mathrm{II}_{1}$ factor given by a subfactor planar algebra is maximal amenable

ARNAUD BROTHIER

Representation theory of type B and C standard Levi $W$-algebras

JONATHAN BROWN and SIMON M. GOODWIN

New invariants for complex manifolds and rational singularities

RONG DU and YUN GAO

Homogeneity groups of ends of open 3-manifolds

DENNIS J. GARITY and DUŠAN REPOVŠ

On the concircular curvature of a $(\kappa, \mu, \nu)$-manifold

Florence Gouli-Andreou and Evaggelia Moutafi

Genuses of cluster quivers of finite mutation type

FANG LI, JICHUN LIU and YICHAO YANG

Taut foliations in knot complements

TAO LI and RACHEL ROBERTS

On the set of maximal nilpotent supports of supercuspidal representations 169

QIN YUJUN

The natural filtrations of finite-dimensional modular Lie superalgebras of 199 Witt and Hamiltonian type

Keli Zheng, Yongzheng ZHANG and WeI Song

Free Brownian motion and free convolution semigroups: multiplicative 219 case

PING ZHONG 